\title{
Nano-formulated curcumin accelerates acute wound healing through Dkk-1-mediated fibroblast mobilization and MCP-1-mediated anti-inflammation
}

\author{
Xinyi Dai ${ }^{1,2}$, Juan Liu ${ }^{1}$, Huaiyuan Zheng ${ }^{1}$, Johannes Wichmann ${ }^{1}$, Ursula Hopfner ${ }^{1}$, Stefanie Sudhop ${ }^{3}$, \\ Carina Prein ${ }^{3}$, Yi Shen ${ }^{4}$, Hans-Günther Machens ${ }^{1}$ and Arndt F Schilling ${ }^{1,2}$
}

Turmeric, a product of Curcuma longa, has a very long history of being used for the treatment of wounds in many Asian countries. Curcumin, the principal curcuminoid of turmeric, has recently been identified as a main mediator of turmeric's medicinal properties. However, the inherent limitations of the compound itself, such as hydrophobicity, instability, poor absorption and rapid systemic elimination, pose big hurdles for translation to wider clinical application. We present here an approach for engineering curcumin/gelatin-blended nanofibrous mats (NMs) by electrospinning to adequately enhance the bioavailability of the hydrophobic curcumin for wound repair. Curcumin was successfully formulated as an amorphous nanosolid dispersion and favorably released from gelatin-based biomimetic NMs that could be easily applied topically to experimental wounds. We show synergistic signaling by the released curcumin during the healing process: (i) mobilization of wound site fibroblasts by activating the Wnt signaling pathway, partly mediated through Dickkopf-related protein-1, and (ii) persistent inhibition of the inflammatory response through decreased expression of monocyte chemoattractant protein-1 by fibroblasts. With a combination of these effects, the curcumin/gelatin-blended NMs enhanced the regenerative process in a rat model of acute wounds, providing a method for translating this ancient medicine for use in modern wound therapy.

NPG Asia Materials (2017) 9, e368; doi:10.1038/am.2017.31; published online 31 March 2017

\section{INTRODUCTION}

Severe acute wounds, as a result of trauma or surgery, are estimated to affect 300 million people around the world every year and the number is still on the rise. This makes wound management a significant and continuous challenge to our healthcare systems. ${ }^{1}$ Noninvasive wound therapy, especially the utilization of plants and their products, has a long history and is still being widely practiced in many cultures. ${ }^{2}$ One example from Asia is the use of turmeric. This plant has been used empirically for nearly 4000 years to cleanse wounds and stimulate their recovery. ${ }^{3}$ The constant search for novel compounds in western medicine has drawn the attention of the scientific community to this ancient remedy. Curcumin was identified as the principle biologically therapeutic compound of turmeric and was found to possess a wide range of beneficial pharmacological properties including anti-inflammatory, anti-oxidant, anti-microbial and anti-cancer activities. ${ }^{4}$ Thus, interest in translating the use of curcumin to the clinic in these areas has increased remarkably over the recent years, leading to a burgeoning number of clinical trials and publications. Curcumin (chemical formula: $\mathrm{C}_{21} \mathrm{H}_{20} \mathrm{O}_{6}$;
(1E,6E)-1,7-Bis(4-hydroxy-3-methoxyphenyl)-1,6-heptadiene-3,5-dione) has also been shown to modulate wound-healing processes. ${ }^{5}$ However, progress in translating these findings into clinical application has so far been impeded due to several physicochemical properties of curcumin, especially its hydrophobicity, instability, poor absorption and rapid systemic elimination. ${ }^{6}$ New developments in material sciences now allow for the removal of some of these obstacles. One option to circumvent unwanted properties is nano-formulation of the pharmaceutical compound through electrospinning. This technique from the textile industry has recently emerged as a novel means to generate nanoscale scaffolds for regenerative medicine and controlled-release systems for therapeutic purposes. For example, we recently nano-formulated emodin by means of electrospinning and increased its solubility, and thereby its function in vitro and in vivo. In addition, electrospinning produces nanofiber structures that closely resemble the architecture of the extracellular matrix (ECM), thereby providing an initial support for the healing process. Here we set out to adapt this approach for curcumin to make its beneficial pharmacological properties accessible for topical application on wounds. As a fiber-forming material,

${ }^{1}$ Faculty of Medicine, Department of Plastic Surgery and Hand Surgery, Klinikum Rechts der Isar, Technische Universität München, Munich, Germany; ${ }^{2}$ Clinic for Trauma Surgery, Orthopaedic Surgery and Plastic Surgery, University Medical Center, Göttingen, Germany; ${ }^{3}$ Center for Applied Tissue Engineering and Regenerative Medicine (CANTER), Department of Applied Sciences and Mechatronics, Munich University of Applied Sciences, Munich, Germany and ${ }^{4}$ Bio-ID Center, School of Biomedical Engineering, Shanghai Jiao Tong University, Shanghai, China

Correspondence: Professor AF Schilling, Clinic for Trauma Surgery, Orthopaedic Surgery and Plastic Surgery, University Medical Center, Robert-Koch-Strasse 40, Briefpost: 37099 Göttingen, Göttingen 37075, Germany.

E-mail: arndt.schilling@med.uni-goettingen.de

Received 15 August 2016; revised 15 November 2016; accepted 12 December 2016 
gelatin was selected, which is a mixture of peptides and proteins acquired by partial hydrolysis and denaturing of the triple-helix structure of collagen, the most abundant protein in the ECM. Recent evidence suggested that gelatin is an attractive polymer for tissue engineering because of its biological origin, which exhibits favorable biodegradability and non-antigenicity. ${ }^{7}$ We recently showed that appropriate crosslinking could improve the mechanical properties of the electrospun gelatin nanofibers to values comparable to those of human skin. ${ }^{8}$ This also prevented its rapid dissolution at temperatures at or above $37^{\circ} \mathrm{C},{ }^{9}$ a crucial condition for topical application.

We hypothesized that curcumin could be loaded into a biomimetic vehicle of electrospun gelatin nanofibers to enhance its solubility and availability and that these mats can be used to improve acute woundhealing processes in vitro and in vivo.

\section{MATERIALS AND METHODS}

\section{Electrospinning and cross-linking}

Gelatin (from bovine skin) and curcumin powder were purchased from Sigma-Aldrich (Munich, Germany). Spinning solutions were prepared by dissolving $1 \mathrm{~g}$ gelatin and $0.1 \mathrm{~g}$ curcumin in $10 \mathrm{ml}$ trifluoroethanol. During the electrospinning process, spinning solution was loaded into a $10 \mathrm{ml}$ syringe with a 20-gauge nozzle $10 \mathrm{~cm}$ away from an aluminum foil-wrapped copper plate as the collector. The feed rate of the syringe pump (Cole-Parmer, Vernon Hills, IL, USA) was set to $1.5 \mathrm{ml} \mathrm{h}^{-1}$ with a DC voltage of $15 \mathrm{kV}$. As-prepared samples were vacuum dried to remove residual organic solvents and underwent a cross-linking process. Briefly, 25\% glutaraldehyde solution was mixed with ethanol $(1 \% \mathrm{~V} / \mathrm{V})$ and transferred to the bottom of a vacuum dryer covered by a porous ceramic plate with the as-prepared nanofibrous mats (NMs) on top. The dryer was vacuumized for $24 \mathrm{~h}$ at $4{ }^{\circ} \mathrm{C}$; then, the cross-linked NMs were rinsed in ultrapure water and dried for $24 \mathrm{~h}$ in a freeze dryer.

\section{Nanofiber characterization}

The morphology and surface texture of the electrospun nanofibers were observed via scanning electron microscopy (JSM-5600LV, JEOL, Japan) at an acceleration voltage of $8-10 \mathrm{kV}$. Before scanning electron microscopy imaging, samples were sputter coated with gold for $50 \mathrm{~s}$ to increase conductivity. The average nanofiber size was determined from a minimum of 50 measurements on a typical scanning electron microscopy image using Image J $1.40 \mathrm{G}$ software (NIH, Bethesda, MD, USA). X-ray diffraction (XRD) spectroscopy was carried out with the aid of an X-ray diffractometer (D/Max-2550PC, RigaKu, Tokyo, Japan) with $\mathrm{Cu} \mathrm{K} \alpha$ radiation in the $2 \theta$ range of $0-60^{\circ}$. The operating voltage and current were kept at $40 \mathrm{kV}$ and $300 \mathrm{~mA}$, respectively, and samples were examined at a scanning rate of $1^{\circ}(2 \theta) \mathrm{min}^{-1}$. Differential scanning calorimetry was performed using an MDSC 2910 differential scanning calorimeter (TA Instruments, New Castle, DE, USA). Samples were heated from 20 to $250{ }^{\circ} \mathrm{C}$ at a heating rate of $10^{\circ} \mathrm{C} \mathrm{min}^{-1}$ under a nitrogen flow rate of $40 \mathrm{ml} \mathrm{min}{ }^{-1}$. A Nicolet-Nexus 670 Fourier transform infrared spectrometer (Thermo Fisher Scientific, Waltham, MA, USA) was used to obtain the Fourier transform infrared spectra of the electrospun nanofibers over a range of 500$4000 \mathrm{~cm}^{-1}$ at a scanning resolution of $2 \mathrm{~cm}^{-1}$. Furthermore, nuclear magnetic resonance ( $\left.{ }^{1} \mathrm{H}-\mathrm{NMR}\right)$ spectra of the chemical structure of pure curcumin and loaded curcumin from NMs were recorded in $\mathrm{CDCl} 3-\mathrm{d} 6$ using a Bruker DRX $400 \mathrm{MHz}$ NMR spectrophotometer. To prepare the sample of loaded curcumin for ${ }^{1} \mathrm{H}-\mathrm{NMR}$ analysis, blended NMs were first dissolved in ultrapure water. Then, the suspension was dialyzed for $72 \mathrm{~h}$ in a dialysis bag with a molecular weight cutoff of $3500 \mathrm{MW}$ to exclude gelatin. Lastly, the remaining suspension was lyophilized to obtain curcumin powder ready for NMR spectroscopy.

\section{In vitro curcumin release profile}

The in vitro dissolution of Cc/Glt NM and free curcumin was examined in $50 \mathrm{ml}$ phosphate-buffered saline (PBS, $\mathrm{pH} 7.4$ ) at $37^{\circ} \mathrm{C}$. At predetermined time intervals, $200 \mu \mathrm{l}$ aliquots were withdrawn for sampling and replaced with an equal volume of PBS to maintain a constant volume. Samples were analyzed using a high-performance liquid chromatography system (Agilent 1200, USA) combined with a quadrupole mass spectrometer (API-4000, AB SCIEX, Redwood City, CA, USA). An analytical column $(4.6 \times 150 \mathrm{~mm}, 3.5 \mu \mathrm{m}$, Agilent Eclipse XDB-C18, Santa Clara, CA, USA) was selected as the separation column and maintained at $45^{\circ} \mathrm{C}$. The mobile phase, with a total rate of $300 \mu \mathrm{min}^{-1}$ in gradient mode, was composed of water and methanol, each containing $0.2 \%$ formic acid. The key parameters for curcumin analysis, the declustering potential, collision energy and collision cell exit potential, were fixed at $-70,-50$ and $-12 \mathrm{~V}$, respectively. The release profile of each sample was performed in triplicate. Average values of the dissolved drug at specified time periods were plotted versus time (h).

\section{Tensile mechanical property of Cc/Glt NM}

The tensile mechanical property of curcumin-loaded nanofibrous scaffolds (cross-linked or non-cross-linked NMs) was examined according to the standard ISO 7198 'Cardiovascular implants-Tubular vascular prostheses' by using a universal material tester (H5K-S, Hounsfield Test Equipment Ltd, Redhill, UK). Briefly, rectangular samples $(10 \mathrm{~mm} \times 50 \mathrm{~mm})$ were cut and subjected to strainstress measurements ( $50 \mathrm{~N}$ load cell at $20{ }^{\circ} \mathrm{C}$ and humidity of $60 \%$ ) at a crosshead speed of $10 \mathrm{~mm} \mathrm{~min}^{-1}$. At least five specimens from each sample were tested to study its Young's modulus and elongation at breaking point.

\section{Cell isolation and culture}

The human fibroblast cell line HS-27 (ATCC CRL-1634) was cultured in Dulbecco's modified Eagle's medium (DMEM, Biochrom, Berlin, Germany) supplemented with $10 \%$ fetal calf serum (FCS) at $37{ }^{\circ} \mathrm{C}$ and $5 \% \mathrm{CO}_{2}$. For subculturing, the cells were washed with PBS and detached by adding $0.25 \%$ trypsin-EDTA solution followed by incubation at $37^{\circ} \mathrm{C}$ until the cells detached. Fresh culture medium was added and the cells were seeded in new culture flasks in a 1:4 ratio. The growth medium was renewed two to three times a week. For most experiments, cells were plated into 6- or 96-well plates (Greiner CELLSTAR, St Louis, MO, USA) in serum-free medium.

Peripheral blood mononuclear cells (PBMCs) were freshly isolated from buffy coats from healthy donors (Institute for Clinical Transfusion Medicine and Immunogenetics Ulm, Ulm, Germany) based on the Ficoll density gradient centrifugation technique. Briefly, heparinized human peripheral blood was diluted with warm $\left(37^{\circ} \mathrm{C}\right)$ PBS (Biochrom) before being carefully layered over Ficoll Paque Plus (Amersham Biosciences, Uppsala, Sweden). After centrifugation at $350 \mathrm{~g}$ for $30 \mathrm{~min}$ without braking, the monocyte-enriched hematopoietic precursors were carefully collected at the interface between the PBS and Ficoll Paque solution. Cells were subsequently counted and resuspended in AIM V Serum Free Medium (Life Technologies, Gaithersburg, MD, USA) at a density of $1 \times 10^{6}$ cells per $\mathrm{ml}$ for direct experiments.

Human macrophage cells MV-4-11 (ATCC CRL-9591) were cultured in DMEM supplemented with $10 \% \mathrm{FCS}$ at $37^{\circ} \mathrm{C}$ and $5 \% \mathrm{CO}_{2}$. Growth medium was renewed every 2 to 3 days. For subculturing, fresh medium was added or replaced to maintain a cell concentration between $1 \times 10^{5}$ and $1 \times 10^{6}$ cells per ml.

\section{Cell viability and metabolism}

HS-27 cells were used to assess the cytotoxicity of electrospun NM based on a procedure adapted from the ISO10993-5 standard test method. Briefly, $6 \mathrm{~cm}^{2}$ of different NM (Cc/Glt NM or Glt NM) were incubated in $1 \mathrm{ml}$ DMEM without serum at $37 \pm 1{ }^{\circ} \mathrm{C}$ for $72 \pm 2 \mathrm{~h}$ to obtain sample extracts. Then, conditioned medium of the NM (CM-NM) was prepared by mixing sample extracts with an appropriate amount of cultured medium (DMEM supplemented with $10 \%$ FCS) for cytotoxicity assays. HS-27 were trypsinized when they reached $80-90 \%$ confluency and then seeded into a 96-well plate at a density of $5 \times 10^{3}$ cells per well. Twenty-four hours after cell seeding, the supernatant was replaced by either CM-Cc/Glt NM or CM-Glt NM. A mixture of equal amounts of DMEM and culture medium was used as the control. The lactate dehydrogenase and water-soluble tetrazolium-1 assays were performed every day according to the manufacturer's instructions (Roche, Mannheim, Germany), and data were collected with a Mithras LB940 Microplate Reader (Berthold Technologies, Bad Wildbad, Germany). 


\section{LIVE/DEAD assay}

HS-27 cells were seeded in a six-well plate at a density of $1 \times 10^{5}$ cells per well. Twenty-four hours after cell seeding, the supernatant was replaced by CM-Cc/Glt NM, CM-Glt NM or control medium. The cells were incubated at $37^{\circ} \mathrm{C}$ and $5 \% \mathrm{CO}_{2}$ for 5 days. For the fluorescence-based dual-color cell LIVE/DEAD assay, calcein AM (green, $E_{\mathrm{X}} / E_{\mathrm{m}}=494 / 517 \mathrm{~nm}$ ) and ethidium homodimer-1 (red, $E_{\mathrm{x}} / E_{\mathrm{m}}=528 / 617 \mathrm{~nm}$ ) were added to the culture every other day according to the manufacturer's instructions (Invitrogen, Karlsruhe, Germany). Images were taken via fluorescent microscope and analyzed via Axiovision software (Carl Zeiss, Oberkochen, Germany).

\section{Cell visualization on the NM}

HS-27 cells were trypsinized and re-suspended in DMEM supplemented with $10 \%$ FCS at a concentration of $1 \times 10^{6}$ cellsper ml. Disk-shaped scaffolds were prepared by sampling the electrospun NM using biopunch $(\varphi=6 \mathrm{~mm}$; Acuderm, Fort Lauderdale, FL, USA). The scaffolds $(n=5)$ and cell suspension $(10 \mathrm{ml})$ were transferred into a $15 \mathrm{ml}$ Falcon tube (Greiner CELLSTAR), which was fixed on a tube rotator (MACS Miltenyi Biotech, Bergisch Gladbach, Germany) and incubated at $37{ }^{\circ} \mathrm{C}$ and $5 \% \mathrm{CO}_{2}$. On days 7 and 14 , samples $(n=5)$ were collected. For live-cell imaging, mitochondria were stained by MitoTracker Deep Red FM (Life Technologies) and nuclei were counterstained using NucBlue Live Ready Probes (blue, $E_{\mathrm{x}} / E_{\mathrm{m}}=360 / 460 \mathrm{~nm}$; Life Technologies) according to the manufacturer's instructions. Before fluorescent microscopy, the samples were cut into smaller pieces and were fixed between two glass slides (SuperFrost Ultra Plus, Thermo Scientific, Braunschweig, Germany)

\section{HS-27 in vitro wound-healing assay (Scratch assay)}

HS-27 cells were seeded at $5 \times 10^{5}$ cells per $\mathrm{ml}$ in culture inserts (Ibidi, Martinsried, Germany). Calcein AM was applied to better visualize and track cells as described above. Once cells reached confluency, the insert was removed and the medium was replaced by CM-Cc/Glt NM, CM-Glt NM or control medium. After $36 \mathrm{~h}$, images were taken and cell migration in the open wound area was quantified (Adobe Photoshop CS5, Adobe Systems, San Jose, CA, USA). The results are expressed as a percentage of the open wound compared to that of the original wound.

\section{HS-27 cytokine profile array}

From this and the following in vitro assay, AIM V Serum Free Medium (Life Technologies, Gaithersburg, MD, USA), instead of the cultured medium (DMEM supplemented with 10\% FCS), was mixed with its extracts of electrospun NM (see cell viability and metabolism) to obtain CM-Cc/Glt NM or CM-Glt NM. They were then added to replace the medium from a sixwell plate with pre-seeded ( $24 \mathrm{~h}$ before) HS- $27\left(1 \times 10^{5}\right.$ cells per well); fresh AIM V Serum Free Medium was used as the control. Subsequently, the cell cultures were incubated at $37^{\circ} \mathrm{C}$ and $5 \% \mathrm{CO}_{2}$ for 5 days before the supernatants being collected and subjected to semi-quantitative cytokine analysis using a Human XL Proteome Profiler Array Kit (R\&D Systems, Minneapolis, MN, USA) according to the manufacturer's instructions. In brief, 102 different cytokines were analyzed in duplicate using spotted capture antibodies, followed by incubation with detection antibodies and chemiluminescent visualization. Membranes were finally exposed to X-ray films for $10 \mathrm{~min}$. The mean (blank corrected, $n=2$ ) pixel density was calculated using Image J software.

\section{Protein quantification}

Cultured supernatants from the cytokine array experiments mentioned above were sampled and analyzed by Quantikine enzyme-linked immunosorbent assay (R\&D Systems) for Dickkopf-related protein-1 (Dkk-1), stromal cellderived factor $1 \alpha($ SDF-1 $\alpha)$, monocyte chemoattractant protein-1 (MCP-1) and thrombospondin-1 according to the manufacturer's instructions. Briefly, supernatants were added to 96 -well plates coated with specific monoclonal antibodies of interest incubated at room temperature for $2 \mathrm{~h}$ before the washing steps. Respective cytokine-specific, luciferase-conjugated antibodies were added to each well, incubated for $2 \mathrm{~h}$ at room temperature and washed again. Substrate solution was then added and incubated for $20 \mathrm{~min}$ in the dark, followed by the addition of $50 \mu \mathrm{l}$ stop solution. The concentrations of cytokines were determined by measuring absorbance at $450 \mathrm{~nm}$ using a microplate reader (Mithras LB940, Berthold Technologies) and plotting the standard curve.

\section{$\boldsymbol{\beta}$-Catenin immunofluorescence and HS-27 migration}

HS-27 cells were seeded at $5 \times 10^{5}$ cells per $\mathrm{ml}$ in culture inserts (Ibidi). Once cells reached confluency, the insert was removed and the medium was replaced by CM-Cc/Glt NM, CM-Glt NM or control medium for $24 \mathrm{~h}$. Cells were then fixed with $4 \%$ formaldehyde for $20 \mathrm{~min}$ and washed twice. Next, $400 \mu \mathrm{l}$ of blocking buffer ( $5 \%$ goat serum in PBS) was added and the cells were incubated for $45 \mathrm{~min}$. Then, primary $\beta$-catenin (D10A8 XP Rabbit mAb, Cell Signaling, Danvers, MA, USA) was added and the cells were incubated overnight at $4{ }^{\circ} \mathrm{C}$, followed by the addition of a fluorochrome-conjugated secondary antibody (Alexa Fluor 568 goat anti-rabbit IgG, Invitrogen) for $1-2 \mathrm{~h}$ at room temperature in the dark. The sample was mounted, and images were recorded with a fluorescent microscope and Axiovision software (Carl Zeiss).

\section{Dkk-1-mediated HS-27 migration assay}

HS-27 migration was measured with a Cytoselect 24-well cell migration kit ( $8 \mu \mathrm{m}$, Colorimetric Format, Cell Biolabs, San Diego, CA, USA) according to the manufacturer's protocol. Briefly, HS-27 cells were seeded in a six-well plate at $1 \times 10^{5}$ cells per well and were treated with $\mathrm{CM}-\mathrm{Cc} / \mathrm{Glt} \mathrm{NM}$ or CM-Glt NM until near confluency. For each assay, $500 \mu \mathrm{l}$ of supernatant of each well was added to the corresponding lower well of the migration plate. In addition, Dkk1 was inhibited via addition of either $10 \mu \mathrm{g} \mathrm{ml}^{-1}$ of Human Dkk-1 antibody (R\&D Systems) or $1 \mu \mathrm{M}$ WAY 262611 (Abcam, Cambridge, UK), a small molecule inhibitor of Dkk-1, to the supernatant. In the next step, $300 \mu \mathrm{l} \mathrm{HS-27}$ cells was added to the upper insert at $1 \times 10^{6}$ cells per ml. After $24 \mathrm{~h}$ of incubation, the inserts were taken out and the non-migratory cells were removed by gently swabbing the interior of the inserts. The inserts were then transferred to a clean well and each was treated for $10 \mathrm{~min}$ with staining solution followed by extraction solution. Finally, $100 \mu \mathrm{l}$ of each reaction solution was transferred to a well of a 96-well plate and measured in a plate reader at $560 \mathrm{~nm}$. For a better visual investigation of fibroblast migration, the cells were also examined under a light microscope.

\section{MCP-1-mediated PBMC and macrophage chemotaxis assay}

The PBMC and macrophage chemotaxis assay was also based on a Cytoselect 24-well cell migration kit ( $8 \mu \mathrm{m}$, Colorimetric Format, Cell Biolabs) according to the manufacturer's protocol as described in the aforementioned HS-27 migration assay, except that in the lower well, MCP-1 was selectively inhibited by adding $5 \mu \mathrm{g} \mathrm{ml}^{-1} \mathrm{MCP}-1$ blocking antibody (Abcam) to the supernatant. Similarly, $300 \mu \mathrm{l}$ newly prepared PBMCs or MV-4-11 (see 'Cell culture and collection' section) was added to the upper insert at $1 \times 10^{6}$ cells per $\mathrm{ml}$ and incubated for $12 \mathrm{~h}$ for the final measurement in a plate reader at $560 \mathrm{~nm}$. For a better visualization of PBMCs and non-adherent MV-4-11 chemotaxis, cells were also observed either via light microscopy (PBMC) or fluorescent microscopy (MV-4-11) using fluorescence-based two-color LIVE/DEAD staining supplemented with NucBlue (Life Technologies) to counter stain the cell nuclei.

\section{Rat skin wound model}

Animal experiments were performed according to the ethical guidelines of Shanghai Jiaotong University. Twelve male Sprague-Dawley rats with initial weights of 250-300 g were used for the experiments. Their backs were shaved and two full-thickness punch biopsy wounds $(6 \mathrm{~mm}$ in diameter $)$ were created in the back of each rat. Directly thereafter, a curcumin/gelatin NM (Cc/Glt NM) or a gelatin NM (Glt NM) of the same size was applied to the wounds, whereas control rats were left untreated. For displacement of the NM to be avoided, all wounds were fixed by sterile Medical Infusion Fixation Paster (RENHE, Hangzhou, China). After 15 days, the animals were killed and the wounds were collected for histological analysis. 


\section{In vivo wound closure analysis}

All wounds were photographed from a standard height at the same intervals over a period of 15 days. Three independent, blinded observers evaluated the wound appearances and measured the wound size from the digital photographs through a pixel-based process of calibration, tracing and area calculation using Adobe Photoshop software (CS5, Adobe Systems). The percentage of wound healing is defined as the quotient of the initial wound area and the wound area after a fixed time interval.

\section{Histology and collagen staining of wound tissue sections}

To collect the tissue samples, an area of $8 \mathrm{~mm}$ in diameter including the complete epithelia margins was excised and embedded into paraffin blocks. Serial sections $(5 \mu \mathrm{m})$ were then taken until reaching the central portion of the wound. These sections were stained routinely with hematoxylin and eosin for histological studies. For collagen detection, sections were stained with PicroSirius red (IHC World, Woodstock, MD, USA) following the manufacturer's protocol. Briefly, deparaffinized sections were first stained with Weigert's hematoxylin for nuclei. After the wash step, slides were stained by PicroSirius red for $1 \mathrm{~h}$ and were washed in two changes of acidified water followed by dehydration and mounting. Fluorescence and polarized (orthogonal polarizing) microscopy was applied to evaluate collagen content and quality. ${ }^{10}$

\section{Macrophage immunohistochemistry of wound tissue sections}

Macrophage infiltration was detected by immunohistochemistry, which was performed using a standard technique with slight modification. In brief, newly prepared paraffin sections were dried overnight at $37^{\circ} \mathrm{C}$ and then at $57^{\circ} \mathrm{C}$ for $8 \mathrm{~h}$. After deparaffinization in xylene and rehydration in a graded series of ethanol, the sections were placed in a $10 \mathrm{mmol}^{-1}$ sodium citrate buffer and boiled for $15 \mathrm{~min}$ for antigen retrieval. Sections were then incubated for $10 \mathrm{~min}$ with $3 \% \mathrm{H}_{2} \mathrm{O}_{2}$ (Carl Roth, Karlsruhe, Germany) to inactivate endogenous peroxidase, followed by a blocking step with $10 \%$ goat serum for $1 \mathrm{~h}$. Subsequently, slides were incubated with monoclonal antibodies against the macrophage marker CD68/ED1 (Abcam) and incubations were carried out overnight at $4{ }^{\circ} \mathrm{C}$. After that, the sections were incubated with a peroxidasecoupled goat anti-mouse antibody for $1 \mathrm{~h}$ and the peroxidase was disclosed using the DAB Substrate Kit (Thermo Fisher Scientific, Darmstadt, Germany). The sections were later counterstained with Mayer's hematoxylin and mounted. Images were taken with an AxioCam camera microscope (PrimoVert, Carl Zeiss). All histological and immunochemical analyses were independently and blindly assessed by three observers, and the images presented are representative of all replicates.

\section{RESULTS}

Curcumin nanoformulation and controlled-release via electrospinning

Addition of curcumin to the electrospun NMs was macroscopically evident through a change in color (Figure 1a). To evaluate the extent to which curcumin was nano-formulated, we first examined the morphology of the NMs by scanning electron microscopy (Figure 1b). The surfaces of both crosslinked and non-cross-linked fibers were smooth without visible aggregates separating from the fiber matrix. Under XRD spectroscopy, the sharp intensive peaks of pure curcumin at $2 \theta$ diffraction angles of $7.96^{\circ}, 8.78^{\circ}, 12.26^{\circ}, 14.54^{\circ}$ and $17.16^{\circ}$ and various peaks of lower intensity indicated that curcumin is present in crystalline form (Figures 1c(a)). Gelatin, on the contrary, is an amorphous polymer having no crystalline structure marked by the absence of any diffraction peak in the range tested for electrospun gelatin NMs (Figures 1c(b)). However, characteristic peaks of crystalline curcumin disappeared completely in the spectrum of blended NMs (Figures 1c(c)), which indicated that after electrospinning, curcumin already lost its crystallinity and then existed as an amorphous nanosolid dispersion, supporting a molecular level dispersion of curcumin in the electrospun nanofiber. Correspondingly, the differential scanning calorimetry curve of pure curcumin exhibited a single sharp endothermic peak at $178.2^{\circ} \mathrm{C}$ by virtue of its melting point (Figures $1 \mathrm{~d}(\mathrm{a})$ ), whereas no such peak was shown in the case of Glt NM (Figures 1d(b)) and Cc/Glt NM (Figures 1d(c)), suggesting that curcumin had molecularly disappeared in the nanofiber as a result of the transition of its physical state, specifically from its original crystalline state to the later amorphous state, which was consistent with the results obtained from XRD studies. We further scrutinized the fibers with Fourier transform infrared spectrometry for an indication of the presence of curcumin in the fibers (Figure 1e). For pure curcumin (Figures 1e(a)), the sharp peak at $3507 \mathrm{~cm}^{-1}$ and a broad one at $3293 \mathrm{~cm}^{-1}$ indicated the presence of $\mathrm{OH}$. The most dominant peaks at 1629 and $1603 \mathrm{~cm}^{-1}$ were attributed to predominantly mixed stretching vibrations of $\mathrm{v}(\mathrm{C}=\mathrm{C}), \mathrm{v}(\mathrm{C}=\mathrm{O})$ and the symmetric aromatic ring stretching vibrations of $\mathrm{v}\left(\mathrm{C}=\mathrm{C}_{\text {ring }}\right)$, respectively. Another characteristic peak at $1510 \mathrm{~cm}^{-1}$ corresponded to highly mixed vibrations of $\mathrm{v}(\mathrm{C}=\mathrm{O}), \delta(\mathrm{CCC})$ and $\delta(\mathrm{CC}=\mathrm{O})$, and aromatic $\mathrm{v}(\mathrm{CC})$ and $\mathrm{v}(\mathrm{CCH})$. Deformation vibrations of the two methyl groups appeared at $1460-1430 \mathrm{~cm}^{-1}$. The band at $1282 \mathrm{~cm}^{-1}$ belonged to the pure in-plane $\mathrm{C}-\mathrm{H}$ vibrations of the aromatic rings. The absorption band at $1275 \mathrm{~cm}^{-1}$ was due to the enol $\mathrm{C}-\mathrm{O}$ stretching. The bands at $1232-1116 \mathrm{~cm}^{-1}$ were assigned to the inplane deformation vibration of phenyl rings and skeletal in-plane deformations, whereas the $\mathrm{C}-\mathrm{O}-\mathrm{C}$ peak was obtained at 1153 and $1024 \mathrm{~cm}^{-1}$. The spectrum of gelatin (Figures $1 \mathrm{e}(\mathrm{b})$ ) showed distinguishing features of the amide A peak ( $\mathrm{N}-\mathrm{H}$ stretching vibration) at $3302 \mathrm{~cm}^{-1}$, amide I peak $\left(\mathrm{C}=\mathrm{O}\right.$ stretch) at $1643 \mathrm{~cm}^{-1}$, amide II peak $\left(\mathrm{N}-\mathrm{H}\right.$ bend and $\mathrm{C}-\mathrm{H}$ stretch) at $1540 \mathrm{~cm}^{-1}$ and amide III peak (C-N stretch plus $\mathrm{N}-\mathrm{H}$ in phase bending) at $1241 \mathrm{~cm}^{-1}$. The spectrum of curcumin-gelatin-blended nanofibers (Figures $1 \mathrm{e}(\mathrm{c})$ ) exhibited features comprising composite characteristic bands of both curcumin and gelatin, such as the mixed vibrations of curcumin at $1510 \mathrm{~cm}^{-1}$ and the $\mathrm{N}-\mathrm{H}$ stretching band $\left(3302 \mathrm{~cm}^{-1}\right)$ or $\mathrm{C}=\mathrm{O}$ stretching band $\left(1643 \mathrm{~cm}^{-1}\right)$ from gelatin. Notably, the characteristic $\mathrm{O}-\mathrm{H}$ absorption peak $\left(3507 \mathrm{~cm}^{-1}\right)$ of curcumin disappeared in the spectrum of Cc/Glt NM, whereas its aromatic signature $\left(1282 \mathrm{~cm}^{-1}\right)$ was apparent. This indicates a successful incorporation of curcumin into gelatin nanofibers and a possible interaction such as an intermolecular hydrogen bond between curcumin and gelatin. To better understand the state of the loaded curcumin, we compared the ${ }^{1} \mathrm{H}-\mathrm{NMR}$ spectra of pure curcumin and curcumin released from nanofibers. As shown in Figure 1f, pure curcumin exhibited proton signals at 3.89 and $6.32-7.39$, which is in agreement with a previous report. ${ }^{11}$ Interestingly, the resonance of loaded curcumin (derived from the dissolution of Cc/Glt NMs) presented at the same chemical shift values (p.p.m.), validating a retained chemical integrity of loaded curcumin in the nanofiber following electrospinning. Next, we evaluated the curcumin release profile in vitro. We found a significantly enhanced curcumin dissolution rate manifested by a sustained releasing pattern in blended NMs compared with that of the insoluble raw curcumin (Figure 1g). Non-cross-linked NMs collapsed immediately upon immersion. Additional cross-linking improved the release kinetics of the entrapped curcumin as well as the mechanical properties of the NMs (3.376 versus $0.367 \mathrm{~N} \mathrm{~cm}^{-2}$, ${ }^{* * * *} P<0.0001$, Figure $\left.1 \mathrm{~h}\right)$.

Fibroblast proliferation and migration are enhanced by Cc/Glt NMs Next, Cc/Glt NMs were tested for their biocompatibility. As fibroblast cells are key factors in wound healing, ${ }^{12}$ we chose to use them for our experiments. Cell cultures of human fibroblast cells (HS-27) treated with supernatants from NMs with or without curcumin did not show different levels of lactate dehydrogenase, suggesting no cytotoxic effect 
a
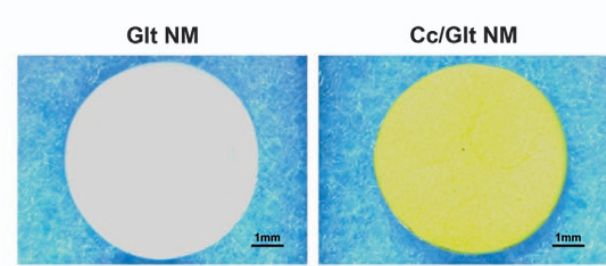

b

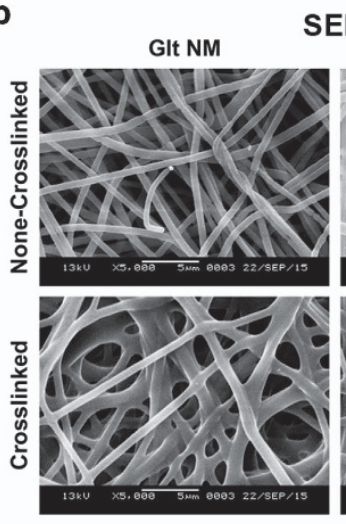

SEM

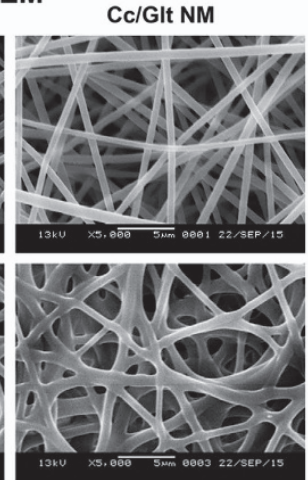

C

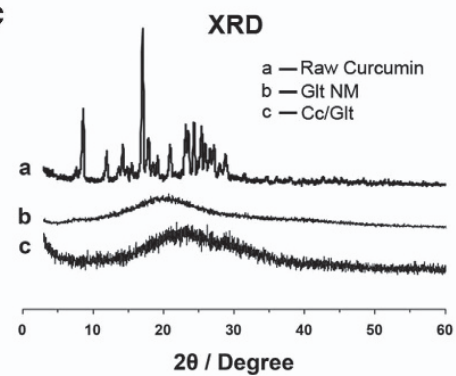

e
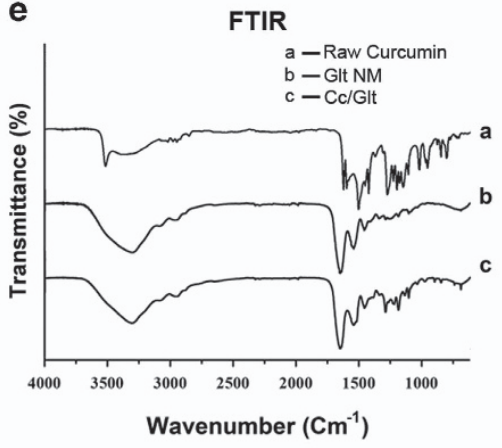

g

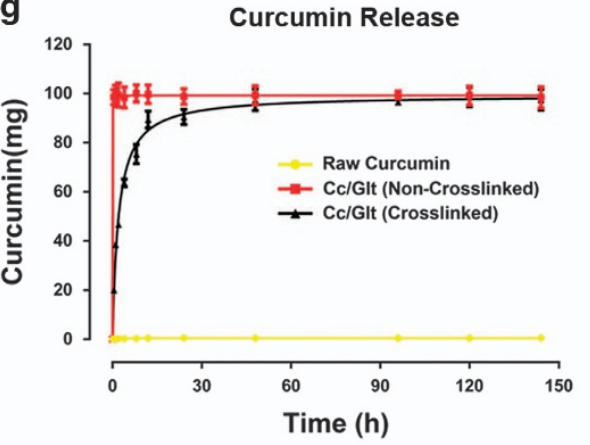

h

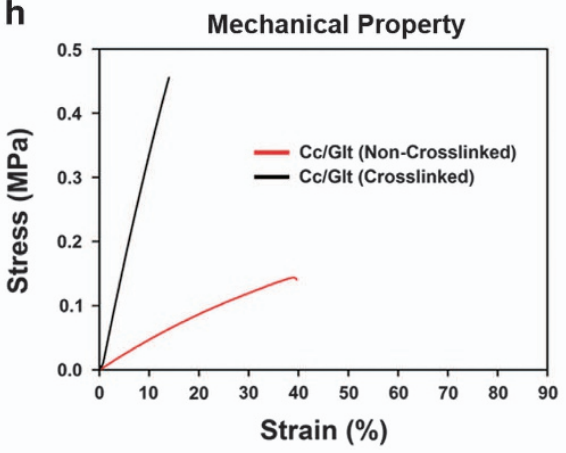

d

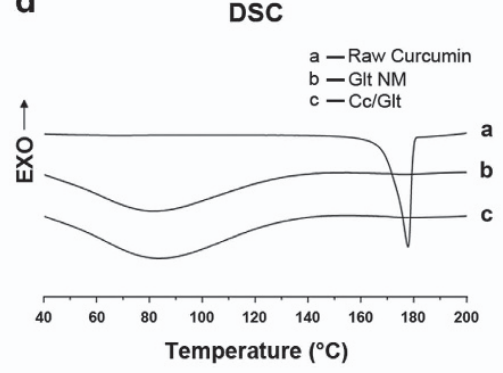

f

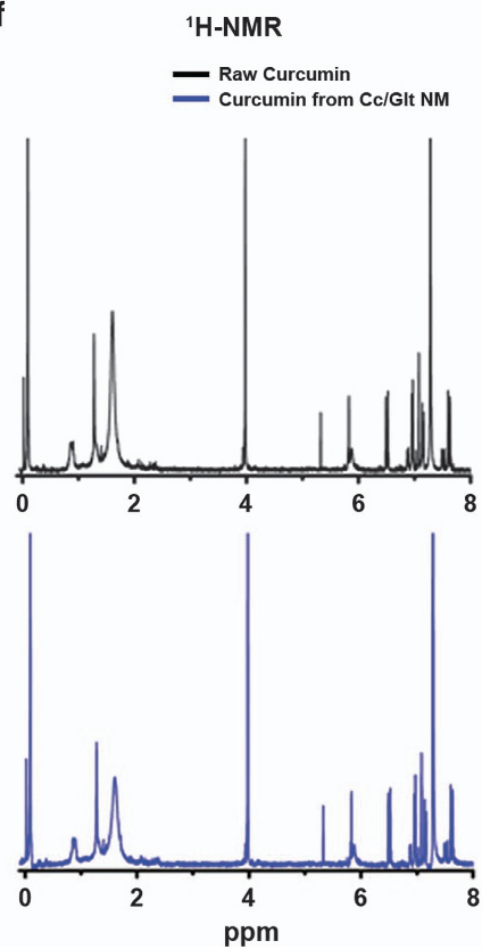

Figure 1 Curcumin nanoformulation and controlled-release via electrospinning. (a) Gross view of the electrospun NM with color change due to curcumin incorporation. (b) Morphology of electrospun nanofibers. Representative scanning electron microscopy images show both crosslinked (lower row) and noncross-linked (upper row) nanofibers exhibiting smooth fiber matrix. Furthermore, curcumin-loaded nanofibers (right column) exist without any visible aggregates, suggesting a nanosolid dispersion derived from a successful electrospinning process without phase separation. (c) Curcumin nanoformulation. XRD spectrum of pure curcumin in the crystalline form exhibiting numerous notable peaks (a), whereas the spectrum of amorphous gelatin with disordered molecular orientation and polymer arrangement is completely absent of any diffraction peaks (b). However, when curcumin is optimally nanoformulated into the gelatin nanofiber through electrospinning, it is completely converted into the amorphous state indicated by the disappearance of its original discrete peaks from the spectrum of the blended nanofibers (c). (d) Formation of amorphous solid dispersion. The differential scanning calorimetry curve of pure curcumin exhibits an endothermic peak (a). However, when crystalline curcumin changed its state into the amorphous solid dispersion through electrospinning, such a peak disappears entirely in the curve of the curcumin-loaded NMs (c), which is similar to that of the amorphous gelatin NMs (b). (e) Curcumin incorporation in gelatin nanofiber. Fourier transform infrared (FTIR) spectrum of pure curcumin (a) shows dominant peaks of 3507, 1629, 1603 and $1510 \mathrm{~cm}^{-1}$, whereas the spectrum of gelatin (b) exhibits typical absorption bands at 3302, 1643, 1540 and $1241 \mathrm{~cm}^{-1}$. The FTIR spectrum of the optimized curcumin formulation through electrospinning (c), however, shows both characteristic peaks $\left(1510 \mathrm{~cm}^{-1}\right)$ and bands $\left(3302\right.$ or $\left.1643 \mathrm{~cm}^{-1}\right)$ of curcumin and gelatin. Notably, the characteristic $\mathrm{O}-\mathrm{H}$ absorption peak $\left(3507 \mathrm{~cm}^{-1}\right)$ of curcumin disappears in the spectrum of Cc/Glt NM, whereas its aromatic signature $\left(1282 \mathrm{~cm}^{-1}\right)$ is apparent, indicating a successful incorporation of curcumin into gelatin nanofibers and a possible interaction between curcumin and gelatin. (f) Retained chemical integrity of curcumin loaded in the electrospun nanofiber. ${ }^{1} \mathrm{H}$ NMR spectra of pure curcumin (black) and loaded curcumin (blue) displaying identical proton signals (p.p.m. values), verifying that the chemical structure of curcumin is preserved in the blended electrospun NMs. (g) Curcumin-controlled release in vitro. A marked improvement in the dissolution rate of curcumin from electrospun Cc/Glt NM relative to that of the pure drug (yellow) is observed and an additional crosslinking process further improves the release kinetics (black), avoiding the initial burst release of curcumin from the non-cross-linked NM (red). (h) Mechanical property of Cc/Glt NM. Typical stress-strain plots of both cross-linked (black) and non-crosslinked (red) electrospun Cc/Glt NM indicate that the cross-linking process significantly enhances the mechanical property of the fabricated NM.

of either the NMs or the entrapped curcumin (Figure 2a). Cell metabolism was significantly increased over time as measured by water-soluble tetrazolium-1 assay in cultures treated with conditioned medium of curcumin/gelatin NM (CM-Cc/Glt NM) when compared with conditioned medium of gelatin NM (CM-Glt NM) and unconditioned medium (Figure 2b), most likely to be reflecting increased proliferation. These results were confirmed by the fluorescent-based dual-color LIVE/DEAD assay, where cell viability under CM-Cc/Glt NM or CM-Glt NM was well maintained as the overwhelming majority of cells gave off green fluorescence (Calcein- 
a

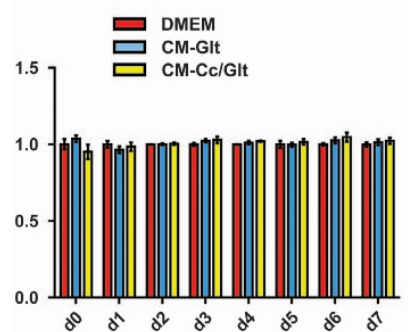

b

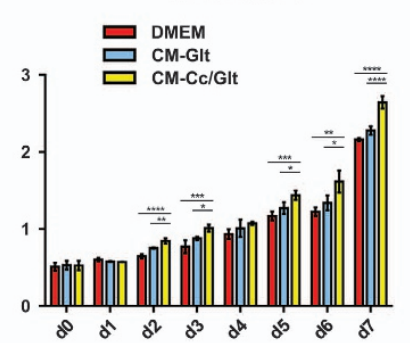

C

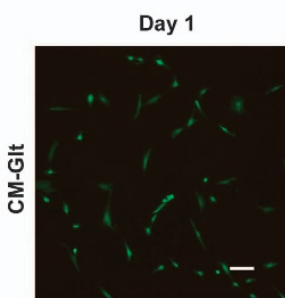

HS-27 LIVE/DEAD assay
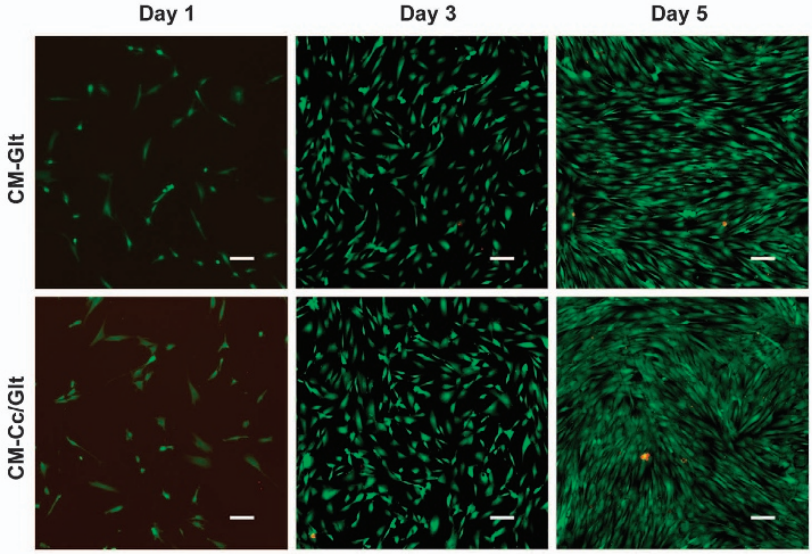

d
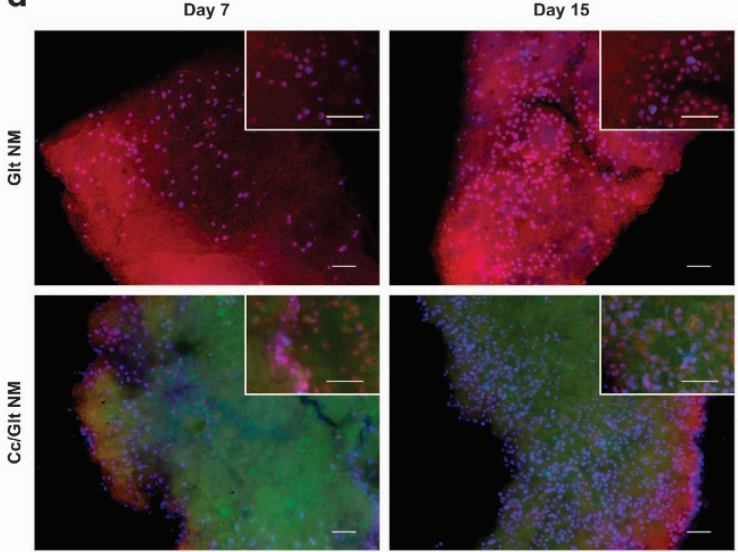

e

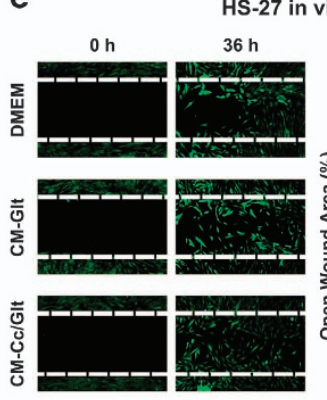

HS-27 in vitro wound healing assay

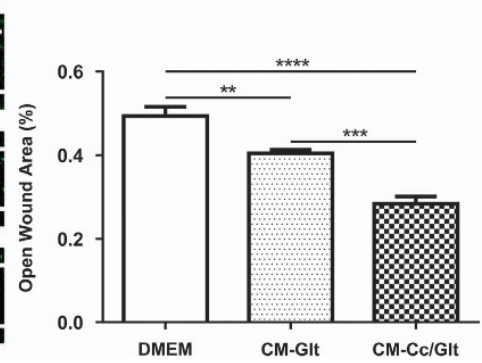

Figure 2 Fibroblast proliferation and migration are enhanced by biocompatible Cc/Glt NM. (a) Human fibroblast cells (HS-27) were treated with conditioned medium of NMs with or without curcumin. Relative cell death was determined by the lactate dehydrogenase (LDH) assay in which the level of LDH release did not change significantly over time. (b) Cell viability (metabolism), quantified by water-soluble tetrazolium-1 (WST-1) assay, was significantly increased upon the treatment of conditioned medium with curcumin. Both assays verify that either fabricated NMs or the entrapped curcumin has no cytotoxic effect on cells. (c) Fluorescence-based cytotoxicity in vitro. HS-27 cells were treated with conditioned medium with or without curcumin for 5 days and evaluated by the fluorescent-based dual-color LIVE/DEAD (viability/cytotoxicity) kit in vitro. No significant differences were observed from scarcely scattered dead cells (red) between the two groups, whereas the overwhelming live cells (green) displayed enhanced cell proliferation over time upon CM-Cc/Glt NM treatment relative to those subjected to CM-GIt NM. (d) Cell visualization on NMs. Live HS-27 cells (nucleus, blue; cytoplasm, red) adhere and expand on the surface of the NMs over time. Differing from Glt NM (upper row), Cc/Glt NM displayed green autofluorescence due to the presence of curcumin in its nanofibers (lower row). Left and right panels show representative images of cell distribution and growth on the mat at different time points, respectively (scale bar $=50 \mu \mathrm{m}$ ). Inserts of higher magnification further illustrate the cell morphology and quantity in detail (scale bar, $50 \mu \mathrm{m})$. (e) Fibroblast in vitro wound healing. HS-27 cells were plated in an Ibidi chamber for $24 \mathrm{~h}$ before scratch wounding by removing the chambers. Cells were cultured for an additional $36 \mathrm{~h}$ and the migration potential was evaluated by measuring the movement of cells into the gap. Representative images were obtained at 0 and $36 \mathrm{~h}$ after the scratch.

AM), whereas dead cells stained red (Ethidium-homodimer-1) were barely found (Figure 2c). Three-dimensional cell adhesion and proliferation experiments further suggested synergistic effects between biomimetic NM and curcumin (Figure 2d). Without curcumin, Glt NM demonstrated cell adhesion and spreading, as observed by the presence of fluorescently labeled live cells (blue nucleus and red cytoplasm) on the Glt NM, suggesting agreeable interactions between cells and Glt NM. Moreover, co-delivering curcumin through Cc/Glt NM exhibited substantially more proliferative cells over time, providing further evidence of curcumin bioactivity together with biocompatibility of Cc/Glt NM for enhanced fibroblast cell activity. Cc/Glt NM exhibited green auto-fluorescence, another piece of evidence of curcumin's $\left(E_{\mathrm{X}} / E_{\mathrm{m}}=420 / 470 \mathrm{~nm}\right)$ presence in the nanofibers.

Next, we investigated in vitro whether activated curcumin delivered by Cc/Glt NM could influence fibroblast migration via the scratch assay. We found that $36 \mathrm{~h}$ after scratching, HS-27 subjected to CM-Cc/Glt NM left the open wound significantly smaller $(28.39 \%$ of the initial gap area) than that from CM-Glt NM (40.44\%, $\left.{ }^{* * *} P<0.001\right)$ and control $\left(49.35 \%,{ }^{* * *} P<0.0001\right)$, suggesting a distinct effect of bioactive curcumin on mobilizing fibroblasts (Figure 2e).

Curcumin-induced fibroblast mobilization is mediated by Dkk-1 To better understand the paracrine mechanism behind the increased migration and proliferation of fibroblasts upon curcumin stimulation, we screened 102 different cytokines, chemokines and growth factors, and identified six significantly downregulated proteins (Figure $3 a$ ). We double checked the secretion of two of the significantly regulated cytokines, Dkk-1 and SDF-1 $\alpha$ by enzyme-linked immunosorbent assay, as recent evidence implies that the SDF-1/CXCR4 axis and Wnt signaling pathway have important roles in fibroblast migration ${ }^{13,14}$ and Dkk-1 is a known Wnt antagonist. Although the decrease in SDF- $1 \alpha$ expression could not be verified, the secreted Dkk1 level was found to be significantly decreased $\left({ }^{* *} P<0.001\right)$ in fibroblasts subjected to CM-Cc/Glt NM (Figure 3b, upper row). To investigate whether curcumin-induced fibroblast migration is through Dkk-1-regulated Wnt signaling, we performed blocking experiments with Dkk-1. In the same scratch assay, we detected 


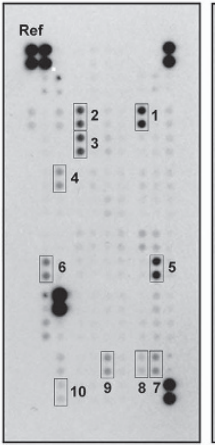

CM-GIt

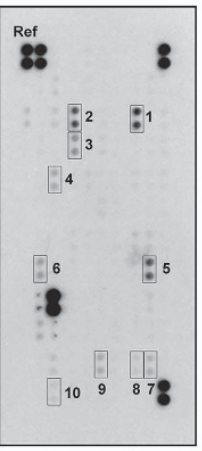

CM-Cc/Glt

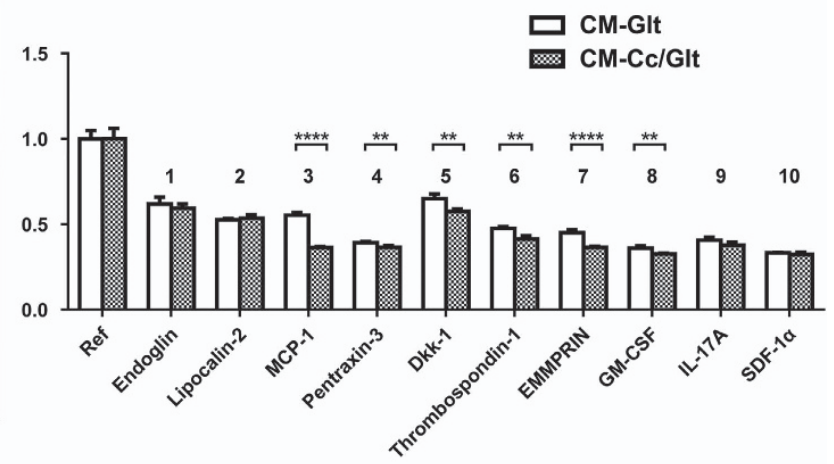

b

Quantikine ELISA assay

Dkk-1 relative expression

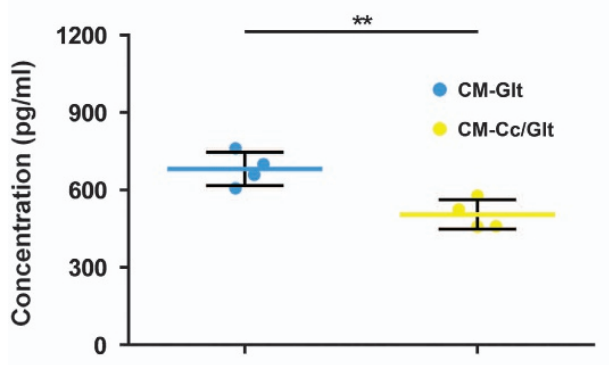

MCP1 relative expression

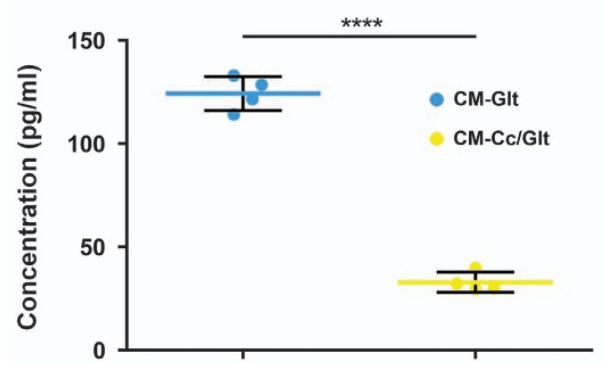

SDF-1 relative expression

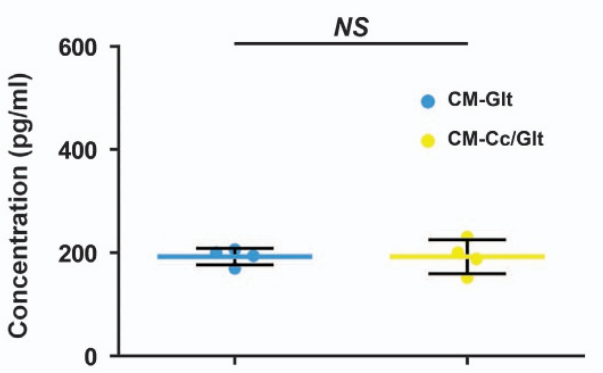

TSP1 relative expression

NS

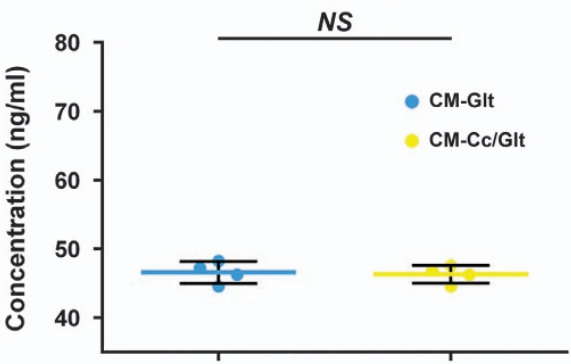

Figure 3 Paracrine profile of fibroblasts upon addition of curcumin. (a) The release of bioactive molecules from HS-27 was evaluated by cytokine protein array. Upon curcumin treatment, several bioactive molecules were found to be significantly downregulated in the conditioned medium with curcumin, including MCP-1, Pentraxin-3, Dkk-1, thrombospondin-1 (TSP-1), EMMPRIN and GM-CSF. Relative secretion was evaluated as pixel intensity relative to that of the positive control. (b) Quantified cytokine expression. Levels of four different cytokines, Dkk-1, SDF-1, MCP-1 and TSP-1, were further analyzed by quantikine enzyme-linked immunosorbent assay from HS-27 subjected to CM-Cc/GIt NM or CM-GIt NM.

immunofluorescence of $\beta$-catenin, the transcriptional co-activator of the Wnt signaling pathway ${ }^{15}$ and observed that migrating cells at the leading edge of wounded fibroblast monolayers displayed evidently stronger $\beta$-catenin signal (Figure $4 \mathrm{a}$, arrow). Treatment with curcumin resulted in enhanced fibroblast migration associated with active $\beta$-catenin accumulation (Figure 4a, first and second rows), which suggested a vital role for Dkk-1 in this process. Selectively blocking Dkk-1 via antibody abrogated the difference between curcumin-treated scratch assays and control; however, it failed to intensify $\beta$-catenin expression (Figure $4 \mathrm{a}$, third and fourth rows). These data were further validated by Boyden chamber-based quantified fibroblast migration assays using either antibody or WAY262611, a small molecule inhibitor of Dkk-1 with high selectivity, which led to exceedingly consistent results (Figure $4 \mathrm{~b}$ ). Such results indicated that Dkk-1 is at least partially involved in the regulation of curcumin- induced fibroblast migration and that a physiological concentration of this cytokine is of critical importance to maintain physiologic fibroblast function.

Curcumin-induced anti-inflammation is mediated through MCP-1 Except for Dkk-1 and SDF-1 $\alpha$, two other cytokines, namely MCP-1 and thrombospondin-1, were also noticeably downregulated in the cytokine array assay described above. Both were previously affirmed as essential participants in wound healing with reportedly common effects pertaining to inflammation. ${ }^{16}$ As an immunomodulatory role of curcumin on wound healing is discussed, ${ }^{5}$ we further investigated whether they may be potential factors here. Again, we first validated the cytokine expression through enzyme-linked immunosorbent assay and found that the MCP-1 level was indeed significantly downregulated $\quad\left({ }^{* * *} P<0.0001\right)$, whereas thrombospondin-1 


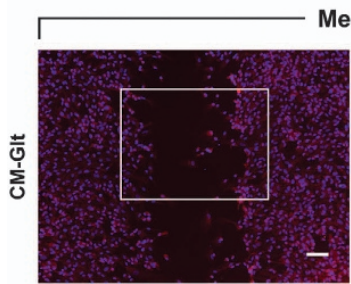

Merge
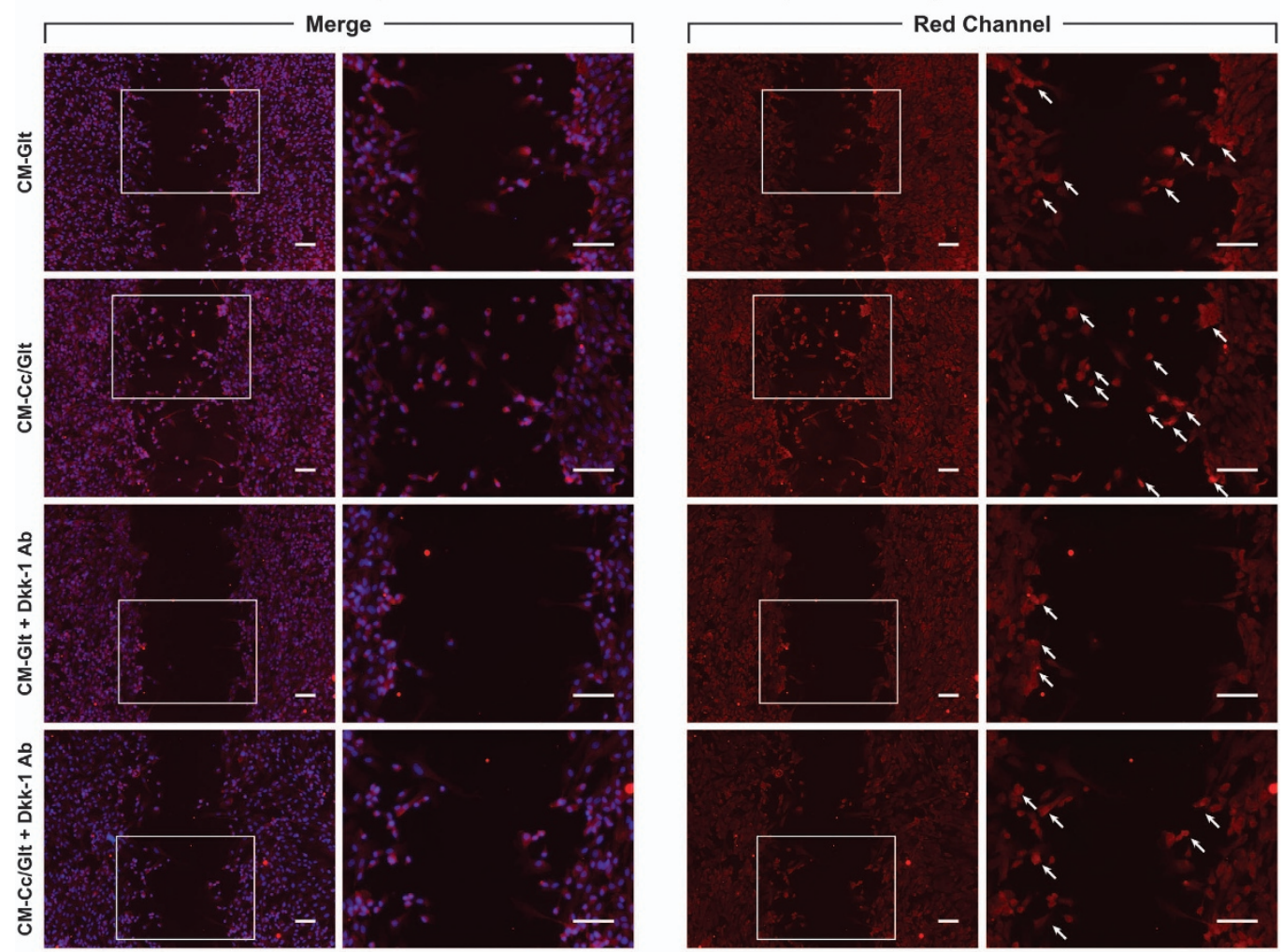

b

HS-27 migration
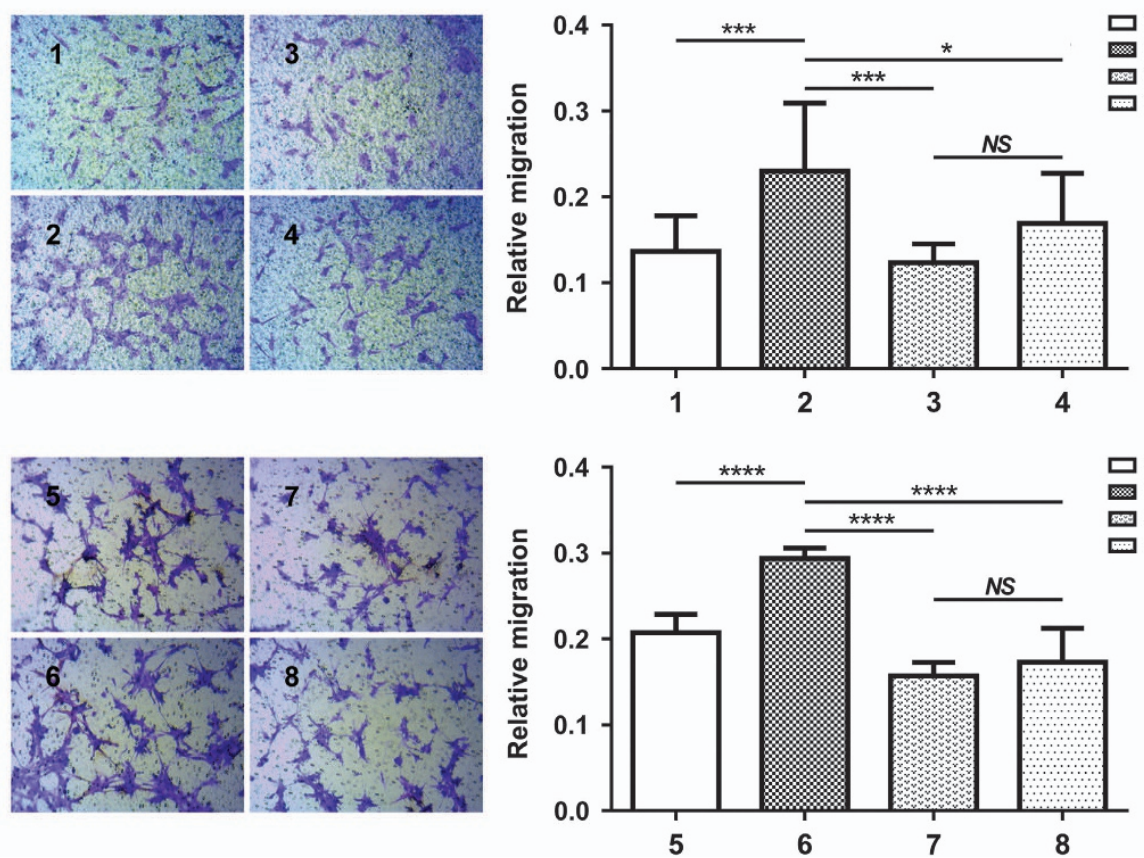

Figure 4 Dkk-1 mediates curcumin-induced fibroblast mobilization. (a) $\beta$-Catenin signaling in migrating fibroblast cells. Fluorescent immunocytochemistry against $\beta$-catenin (red) with nuclei counterstain (blue) in HS-27 cells undergoing a scratch assay $24 \mathrm{~h}$ post wounding are shown (left panel). A single red channel image (right panel) was extracted to emphasize $\beta$-catenin expression, where migrating HS-27 cells at the leading edge of the scratch wound return to an active $\beta$-catenin signal (arrow). Moreover, cells subjected to CM-Cc/GIt NM result in enhanced fibroblast migration associated with active $\beta$-catenin accumulation relative to that of CM-GIt NM (first and second rows, arrow). Dkk-1 neutralization via antibody further abrogated such a difference but failed to intensify $\beta$-catenin expression (third and fourth rows, arrow). (b) Dkk-1 mediates fibroblast migration. Quantified HS-27 migration under Dkk-1 mediation was analyzed by Boyden chamber assay using either Dkk-1 antibody (1-4) or WAY262611 (5-8). Curcumin existence led to significantly enhanced HS-27 migration ( 2 to 1 and 6 to 5). Blockade of Dkk-1 abrogated the difference in curcumin-induced HS-27 migration by both Dkk-1 antibody (4 to 3 ) and its small molecule inhibitor (8 to 7 ). Scale bar $=100 \mu \mathrm{m}$. 
a

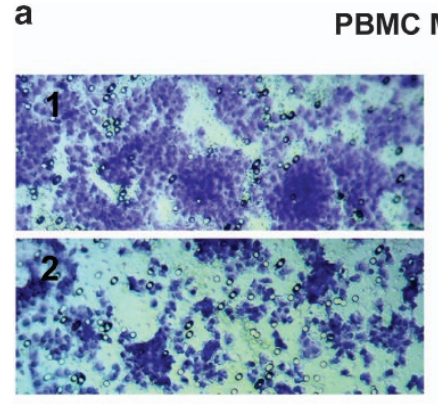

PBMC Migration

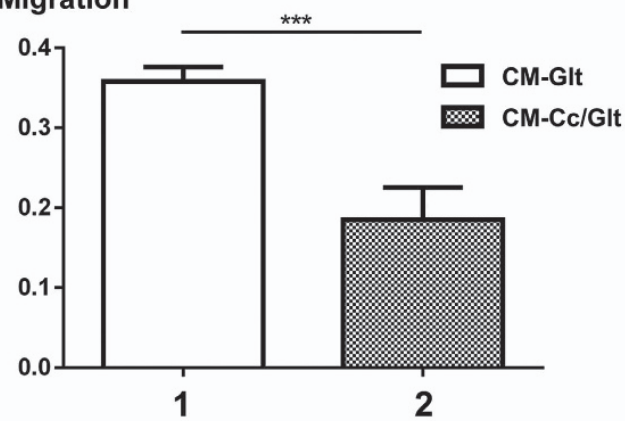

b

MV-4-11 Migration
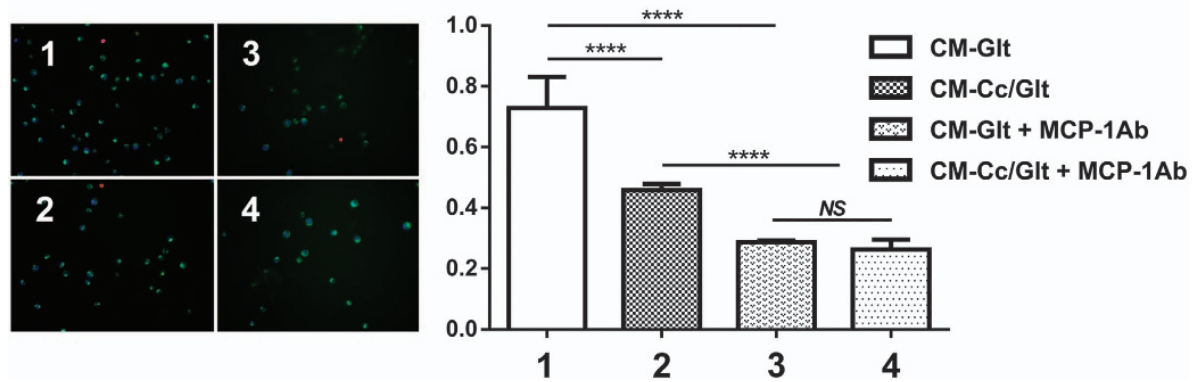

Figure 5 MCP-1 mediates curcumin-induced anti-inflammation. (a) Curcumin inhibits PBMC chemotaxis. The supernatant of fibroblasts subjected to conditioned medium with or without curcumin was added to the lower well of the migration plate and served as the chemoattractant, and newly prepared PBMCs were added to the upper insert and allowed to undergo chemotaxis for $12 \mathrm{~h}$, which is found to be significantly decreased upon curcumin treatment (right, 2 to 1). Chemotactic PBMCs crossing to the opposite site of the membrane were further stained for better visualization (left). (b) Curcumin-inhibited macrophage (MV-4-11) chemotaxis is mediated by MCP-1. Supernatants of fibroblasts subjected to conditioned medium with or without curcumin were added to the lower wells of the migration plate and served as the chemoattractant, and macrophages (MV-4-11) were added to the upper insert and allowed to undergo chemotaxis for $12 \mathrm{~h}$. The existence of curcumin significantly reduced MV-4-11 chemotaxis (right, 2 to 1 ); however, the addition of MCP-1 antibody abrogated the difference (right, 4 to 3). Chemotactic MV-4-11 across the membrane to the lower well was further fluorescently stained (dead cell, red; live cell, green; and cell nucleus, blue) for better visualization (left).

expression remained unchanged when fibroblasts were subjected to $\mathrm{CM}-\mathrm{Cc} / \mathrm{Glt} \mathrm{NM}$ (Figure 3b, bottom row). As MCP-1 is widely accepted as one of the key chemokines that regulates migration and infiltration of monocytes/macrophages to inflammatory sites due to tissue injury or infection, ${ }^{17}$ we then selectively blocked MCP-1 with antibodies in an in vitro functional chemotaxis assay using both PBMCs and a macrophage cell line (MV-4-11) to test whether MCP-1 mediates their chemotaxis in the presence of curcumin. PBMCs in the upper insert indeed demonstrated a significantly diminished migration towards the lower well when the latter contained conditioned medium with curcumin than without curcumin $\left({ }^{* *} P<0.001\right.$, Figure 5a). Similarly, an evident inhibitory effect of curcumin on MV-4-11 chemotaxis was observed as well and, furthermore, this effect could be abrogated when exogenous MCP-1 antibody was applied ( ${ }^{* * *} P<0.0001$, Figure 5 b). It should be noted that the elimination of curcumin-induced changes in PBMC chemotaxis could not likewise be achieved by using MCP-1 antibody in our attempt. This is likely to be due to the multiple cellular components in the isolated PBMCs from the buffy coat, which tend to conceal the effect of one single cytokine from a designated cell type. Nevertheless, highly consistent results obtained from both PBMCs and macrophages indicated that MCP-1 is a pivotal downstream mediator of the curcumin-induced anti-inflammatory response manifested by impeded chemotaxis of inflammatory cells, typically macrophages, in the healing process. Presumably, such an impediment acts throughout the healing process given that MCP-1 originated from mobilized wound site fibroblasts. This was later confirmed by in vivo experiment (see below).

\section{Wound healing in vivo}

We finally evaluated in vivo whether this approach of curcumin nanoformulation by engineering electrospun Cc/Glt NM could be used to enhance wound healing. We used Sprague-Dawley rats and treated full-thickness back-skin wounds of these rats (two wounds per rat, $n=12$ rats) with Glt NM, Cc/Glt NM or left untreated (control). Wound closure was analyzed after 3, 7, 11 and 15 days (Figure 6a). Although wounds that received Cc/Glt NM did not differ from wounds treated with Glt NM on early days post-wounding $\left({ }^{\star} P<0.05\right.$ compared with the control group on day 3$)$, the delivery of Cc/Glt NM led to significantly faster wound closure at time-points of day $7\left({ }^{* * * *} P<0.0001\right)$, day $11 \quad\left({ }^{* * *} P<0.0001\right)$ and day 15 ( ${ }^{* * *} P<0.0001$ compared with the control group and ${ }^{* * *} P<0.001$ compared with the Glt NM group). Furthermore, representative wound histology (hematoxylin and eosin) for all three groups at the final time point (day 15) showed clear differences in the extent of re-epithelialization and the granulation tissue between wounds subject to Cc/Glt NM and other conditions (Figure 6b). Specifically, complete re-epithelialization and differentiated epithelium characterized by well-developed epidermis layers were shown in wounds subjected to $\mathrm{Cc} / \mathrm{Glt} \mathrm{NM}$ (Figures $6 \mathrm{~b}(\mathrm{c}, \mathrm{f}, \mathrm{i})$ ), which clearly differed from less-differentiated epidermis in wounds treated with Glt NM (Figures $6 \mathrm{~b}(\mathrm{~b}, \mathrm{e}, \mathrm{h})$ ) or epithelial incompletion accompanied by fibrinous debris in wounds of the control group (Figures $6 \mathrm{~b}(\mathrm{a}, \mathrm{d}, \mathrm{g})$ ). 

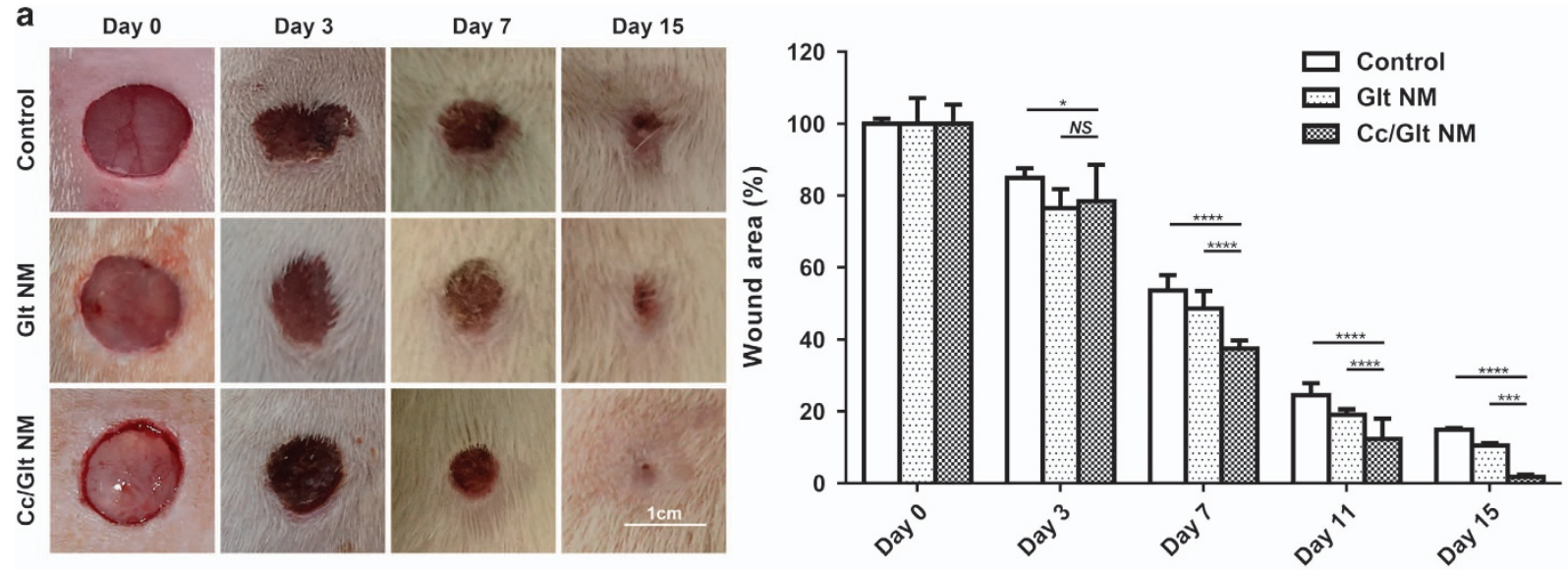

b

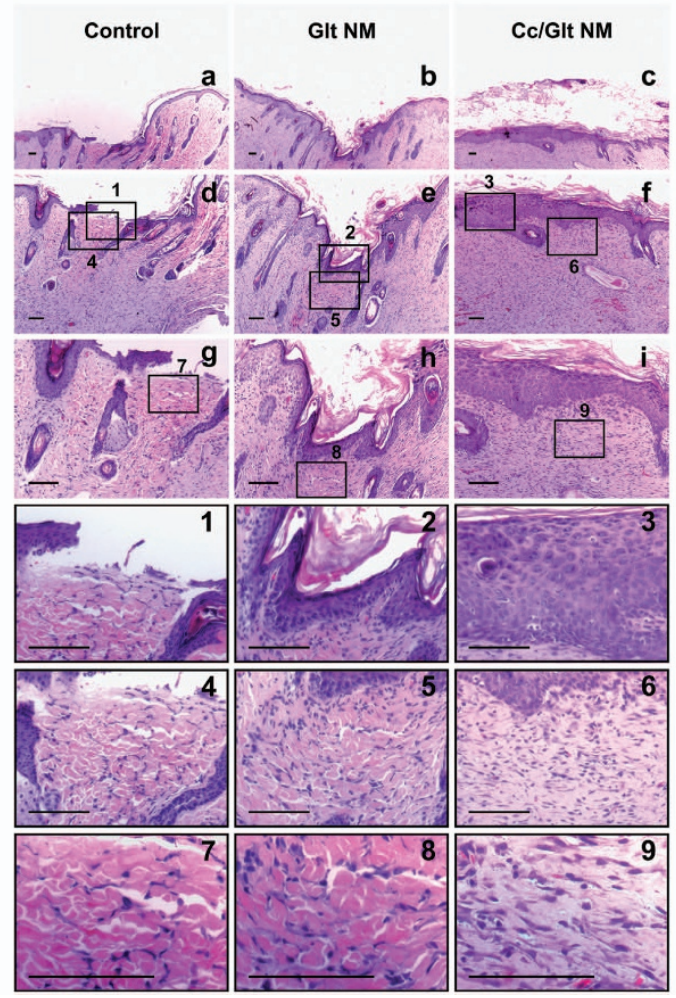

c

Control
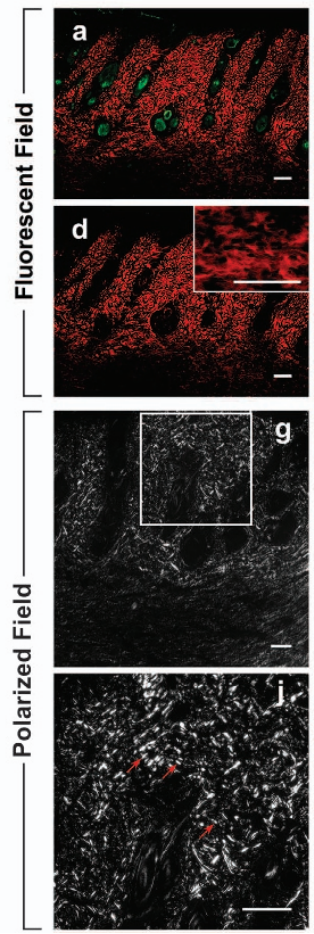

GIt NM
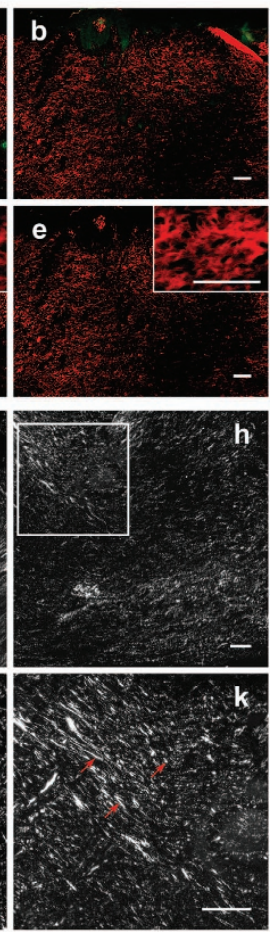

Cc/GIt NM
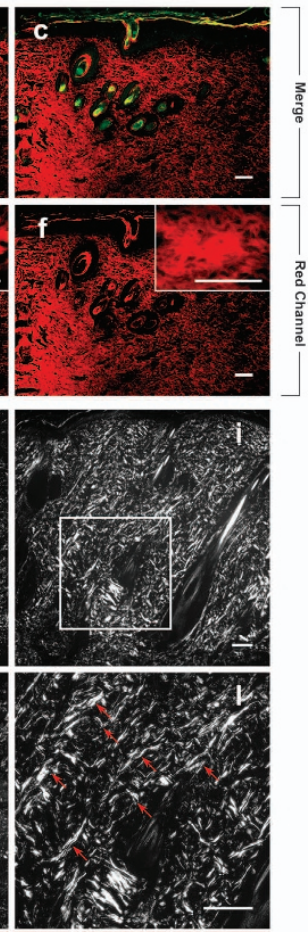

Figure 6 Topical application of Cc/Glt NM accelerates wound healing in vivo. (a) Cc/Glt NM accelerates wound closure. A rat model of acute wounding was used to evaluate the healing ability of Cc/Glt NM in which three groups were tested: wounds treated with Cc/Glt NM or Glt NM only or without treatment (control). Curcumin delivered from the Cc/Glt NM was shown to markedly accelerate wound healing, especially after 3 days post wounding. (b) Cc/Glt NM enhances dermal regeneration. Histological evaluation of repaired skin stained with hematoxylin and eosin 15 days post wounding was performed to compare the rate of re-epithelialization and granulation tissue formation from three different groups: wounds treated with Cc/GIt NM (c, $f$ and i,3,6,9) or with Glt NM only ( $a, d$ and $g, 1,4,7$ ) or without treatment (control, b, e and h,2,5,8). Different levels of re-epithelialization and epithelium differentiation are shown in further detail with higher magnification images (1-3). Furthermore, varying degrees of the maturation of granulation tissue (pink-violet) are shown in detail in images with higher magnification (4-9), both of which demonstrate the superior performance of Cc/Glt NM over Glt NM and the control in improving dermal regeneration for accelerated wound healing (scale bar=100 $\mu \mathrm{m}$ ). (c) Cc/Glt NM enhances collagen deposition. PicroSirius red staining was used to detect the content and quality of newly deposited collagen in the wound bed by fluorescent (a-f) or polarized ( $\mathrm{g}-\mathrm{l})$ microscopy. Collagen (red) is in clear contrast to cells (autofluorescence green) in the merged image (a-c). To focus only on collagen content, a single red channel image (d-f) is used to compare the relative fluorescent intensity with more detail shown in the higher magnification inserts ( $d-f$, upper right corner). The quality of the collagen bundles was further evaluated using polarized light to detect birefringence. Wounds subjected to Cc/Glt NM have brighter and thicker with better curved and more regularly aligned collagen bundle structures (I, red arrow) as compared with those of wounds treated with Glt NM ( $k$, red arrow) or no treatment (j, red arrow). Scale bar=100 $\mu \mathrm{m}$.

Moreover, there was noticeable fibroblast proliferation accompanied by markedly increased deposition of connective tissue in the wounds of the $\mathrm{Cc} / \mathrm{Glt}$ NM-treated group relative to those in the other two groups (Figures $6 \mathrm{~b}(4-9))$. These results indicated a superior performance of $\mathrm{Cc} / \mathrm{Glt} \mathrm{NM}$ over those of Glt $\mathrm{NM}$ and control in healing the wound.

Collagen deposition is another crucial step in granulation tissue formation within the wound bed. Hence, through PicroSirius red 

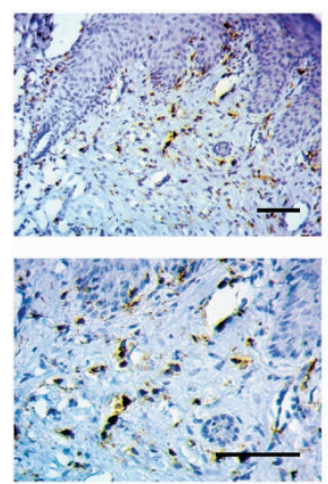

Control
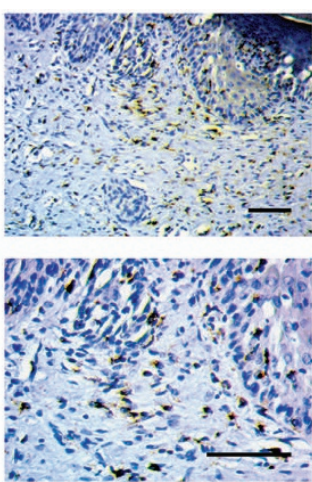

GIt NM
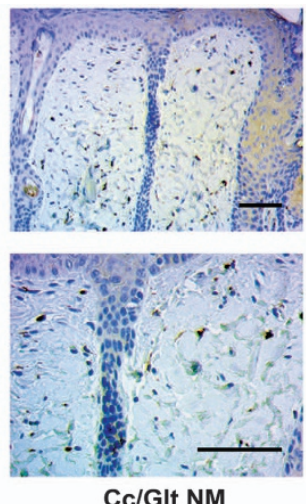

Cc/GIt NM

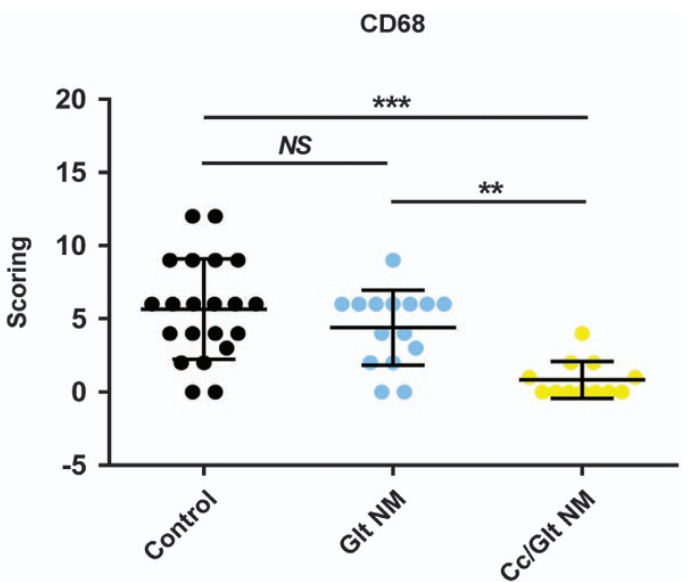

Figure 7 Topical application of Cc/Glt NM inhibits macrophage infiltration in vivo. Expression and localization of CD68 (macrophage marker) in wound beds were examined by immunohistochemistry. Representative photographs from different treatments are shown (left: upper row, low magnification; lower row, high magnification). Macrophage infiltration was quantified by morphometry (right).

staining, we evaluated the extent to which the collagen content and quality differed between the treatments. As shown in Figure 6c, merged fluorescence microscopic images demonstrated a clear distinction between cells (green auto-fluorescence) and extracellular collagen fibers (PicroSirius red -stained red fluorescence, Figures $6 c(a-c)$. To focus only on collagen deposition, a single red channel was used for evaluation Figures $6 \mathrm{c}(\mathrm{d}-\mathrm{f})$ in which $\mathrm{Cc} / \mathrm{Glt}$ NM-treated wounds displayed more collagen deposition as evidenced by a higher density and intensity of PicroSirius red signal (Figure $6 \mathrm{c}(\mathrm{f})$ ) relative to those of wounds subjected to other conditions (Figures $6 c(d, e)$ ). Moreover, this collagen was more compact and better interwoven (Figure $6 \mathrm{c}(\mathrm{f})$ ), obviously differing from the loose reticular arrangement of collagen formed in Glt NM-treated (Figure $6 \mathrm{c}(\mathrm{e})$ ) or untreated (Figure $6 \mathrm{c}(\mathrm{d})$ ) wounds, indicating the formation of a more mature collagen. These findings were further confirmed by polarized microscopy (orthogonal polarizing field), as collagen fibers in Cc/Glt NM-treated wounds (Figures $6 \mathrm{c}(\mathrm{i}, \mathrm{l})$ ) appeared to be not only brighter and thicker than those from wounds receiving Glt NM (Figures $6 \mathrm{c}(\mathrm{h}, \mathrm{k})$ ) or left untreated (Figures $6 \mathrm{c}(\mathrm{g}, \mathrm{j})$ ) but also presented well-curved and more regularly aligned collagen bundle structures that are clearly in contrast to what was found in wounds from the other two groups (Figures $6 c(j-1)$, red arrow). To confirm the in vitro data of curcumin-induced anti-inflammatory response, CD68/ED1 immunohistochemistry was performed to investigate interstitial macrophage infiltration on tissue samples collected at the final time-point (day 15) in a rat model of acute wounds, where a marked decrease in CD68/ED1-positive macrophage influx in interstitial areas was seen in Cc/Glt NM-treated wounds as compared with those from Glt NM-treated $\left({ }^{* *} \mathrm{P}<0.01\right)$ or -untreated (control, ${ }^{* *} \mathrm{P}<0.001$ ) wounds (Figure 7 ).

\section{DISCUSSION}

Currently, there are a number of clinical trials showing curcumin's efficacy against a variety of human diseases with few side effects. ${ }^{18}$ However, clinical use in wounds, although discussed, has so far been restricted mainly because of the low bioavailability of curcumin due to its feeble solubility. For an optimized wound therapy with curcumin, a suitable carrier system would be necessary to deliver therapeutic doses of curcumin ideally as wound dressing. In the current study, we demonstrate such a system that allows for considerably enhancing the solubility and availability of curcumin by embedding it into gelatinbased electrospun NMs. With regard to the state of loaded curcumin in the electrospun nanofibers, XRD and differential scanning calorimetry are crucial for examining the dispersion and crystallinity of curcumin in the polymer matrix. The absent XRD crystalline peaks attributable to curcumin in our fabricated system revealed that curcumin crystals were transformed to an amorphous state (Figure 1c). Furthermore, the disappearance of thermal features of curcumin (Figure 1d) suggests some interaction between curcumin and gelatin, most likely due to the formation of an amorphous solid dispersion, a known condition for increasing drug dissolution. ${ }^{19}$ On the other hand, the hydrogen bonding between curcumin and gelatin, verified by Fourier transform infrared spectroscopy (Figure 1e), would inhibit curcumin crystallization and let the compound precipitate out in the amorphous form, resulting in increased curcumin solubility. Hence, it is likely to be that gelatin inhibited the association of curcumin molecules to form crystal nuclei and subsequent crystal growth in the nanofibers during the electrospinning process. Most importantly, however, the chemical structure of curcumin was preserved throughout the entire process (Figure 1f), which is of crucial importance to exert its biological effects after being released.

We chose gelatin as the fiber-forming material because of its biological origin from its parent collagen, the principal structural element of skin ECM. It contains the Arg-Gly-Asp-like sequences, which are essential for cell adhesion and migration in wound healing. ${ }^{20}$ In addition, it has been found as a repository for a large family of cytokines. ${ }^{21}$ Thus, the non-woven electrospun nanofibers could resemble ECM structures to provide mechanical protection as well as nano-topography and chemical signaling in the guidance of cell function and also serve as a biodegradable scaffold for in situ dermal regeneration. Moreover, gelatin has excellent water absorption and fluid affinity, supporting moist wound healing. Several in vitro assays demonstrated that our approach endowed the biomimetic construct with appropriate properties necessary for fibroblast growth and activity similar to what granulation tissue ECM would do in physiological wounds. We also observed that the nanoscale pores presented in our electrospun NMs avoided cell infiltration and tissue ingrowth (data not shown).

This is in accordance with previous reports that electrospun nanofibrous meshes as wound dressings are easily removable from the wound without trauma (tissue ingrowth) and protect against microorganisms from the external environment while allowing gas and fluid exchange through inherent small interstices. ${ }^{22}$ Considering 


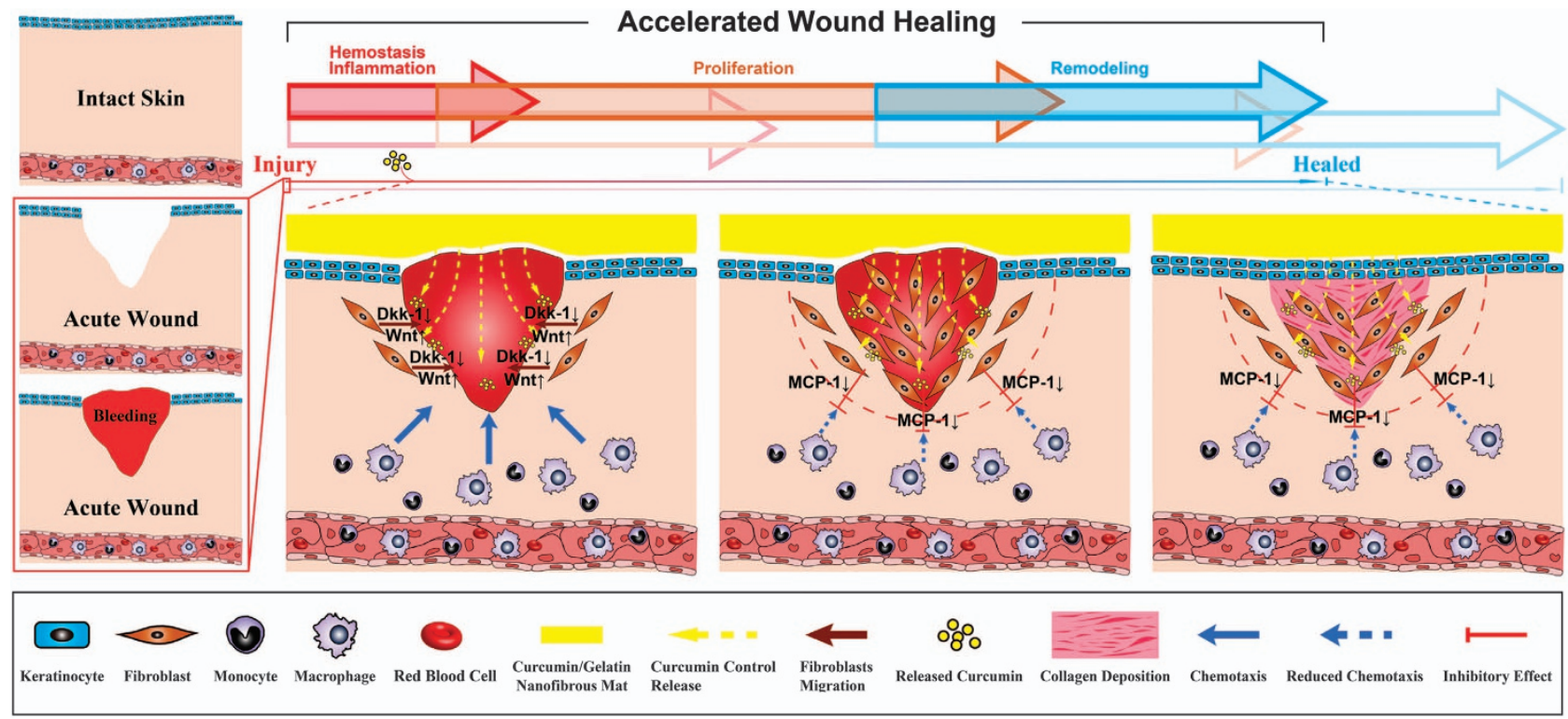

Figure 8 Scheme of the potential mechanism for curcumin-induced accelerated wound healing. Upon injury, topically applied Cc/Glt NM leads to controlled release of bioactive curcumin, which in turn mobilizes wound site fibroblasts through the enhancement of both cell proliferation and migration, a process partially mediated by Dkk-1-regulated Wnt/ $\beta$-catenin pathway signaling. In the meantime, fibroblasts decrease the expression of MCP-1, which mediates monocyte/macrophage chemotaxis, leading to lasting inhibition of the inflammatory response during the healing process. As a result, the wound environment turns out to be more favorable for later stages of wound healing with faster re-epithelialization, granulation tissue formation, as well as collagen deposition and maturation from proliferative fibroblasts.

the fact that wound dressings also need to serve as a mechanical support and barrier while covering the wound, an appropriate tensile strength of the mat is necessary. We took additional cross-linking steps to engineer the modulus of elasticity of $\mathrm{Cc} / \mathrm{Glt} \mathrm{NM}$ into the range reported for normal human skin, which has been thought to be crucial for wound dressings and skin replacement constructs. Most importantly, this changed the release kinetics for curcumin from the NMs to a time window that conforms with accepted clinical dressing change intervals. ${ }^{23}$ Together with gelatin's wide commercial availability at relatively low cost, the easily produced Cc/Glt NM is therefore a promising candidate for clinical translation.

Delivered curcumin was shown to recruit wound site fibroblasts in vitro and in vivo (Figures $6 \mathrm{~b}$ and 8 ) by inducing proliferation and migration (Figures 2,6b and 8), which is at least partially mediated by Dkk-1 (Figure 4). Our results agree with those of a previous report showing decreased expression of Dkk-1 and enhanced expression of $\beta$-catenin in fibroblasts from pachydermoperiostosis (abnormal fibroblast proliferation) patients. ${ }^{24}$ Similarly, the Dkk-1-mediated Wnt signaling pathway has also been found as a positive regulator of cell migration in other tissue such as breast cancer. ${ }^{25}$ Interestingly, however, Koch et al. ${ }^{26}$ found that wounding of epithelial monolayers returned the leading edge cells to a nonquiescent state with activated $\beta$-catenin signal but subsequent induction and secretion of Dkk-1. Given that the Wnt signaling cascade with its antagonist Dkk-1 has been proposed to have crucial roles for maintaining homeostasis of a variety of tissues including the skin, ${ }^{27}$ and that the physiologic Dkk-1 level is of vital importance to maintaining its biological functions, ${ }^{28}$ we speculate that curcumin could precisely control the Dkk-1 level in a temporospatial manner to regulate the downstream Wnt signaling pathway, as Dkk-1 has been shown to participate in a negative feedback loop in Wnt signaling as well. ${ }^{29}$ However, more studies are required to fully elucidate its less well-understood role in wound healing.
Furthermore, curcumin action on fibroblasts also leads to inhibition of inflammatory triggers by suppressing MCP-1 levels and subsequent inflammatory cell infiltration in vitro and in vivo. We proved that such inhibition could exist lastingly through the entire healing process, not just in the inflammatory phase, which is complementary to the current knowledge. ${ }^{30}$ Presumably, this is beneficial for the formation and maturation of granulation tissue, because it protects the newly formed granulation tissue from degradation by proteases, in particular the matrix metalloproteinases (MMPs), a family of zinc-dependent, calcium-containing endoproteinases produced by immune cells such as macrophages and neutrophils. Studies have also shown that the prolonged exposure of wound tissues to pro-inflammatory cytokines, for example, interleukin and tumor necrosis factor- $\alpha$, may act to stimulate the production of MMPs, while inhibiting the synthesis of tissue inhibitors of metalloproteinases. ${ }^{31}$ Furthermore, MCP-1 has recently been shown to up-regulate MMP-1 and MMP-2 mRNA expression in human skin fibroblasts in which interleukin- $1 \alpha$ has been found to take part as a mediator through an autocrine loop. ${ }^{32}$ As a matter of fact, curcumin itself possesses distinct chemical properties that allow it to use the metal atoms of metalloproteins as a means to interact with these proteins and become a natural MMP inhibitor. Such ability of multivalent metal chelation lies on curcumin's central moiety that can complex metals at its $\beta$-dicarbonyl oxygen atoms (diketo form) or ketone oxygen (keto-enol form), which has a high negative charge. Thus, it forms chelates with catalytic zinc ions in the active sites of MMPs and turns out to be a potent broad-spectrum inhibitor. ${ }^{33}$ The molecular docking studies revealed that curcumin coordinated with zinc atoms and appeared to bind in an extended conformation making extensive van der Waals contacts and hydrogen bonding, as well as $\pi-\pi$ interactions with key residues in the active site of the enzyme, demonstrating similar binding affinities and modes as those of known inhibitors of MMPs. ${ }^{34,35}$ Needless to say, such a biological process could only be facilitated through enhanced bioavailability of curcumin. All of these pieces of evidence 
are consistent with the observation that the improved response in vivo is only evident in the later stages of wound healing. Faster mobilization of fibroblasts in combination with inhibition of degrading cells would lead to an earlier maturation of the granulation tissue that can then be more quickly and better re-epithelialized by the surrounding epithelium. The presence of persistent inflammatory stimuli leading to a prolonged inflammatory response consisting of neutrophils and macrophages is a characteristic associated with impaired wound healing, for example, among patients with diabetes. Consequently, the engineered Cc/Glt NM may also be suitable for problematic wounds such as diabetic chronic ulcers. More work is needed here for the application of the medicated NMs to some advanced animal models of chronic/impaired wound healing, such as on streptozotocin-induced hyperglycemic diabetic mouse models or pig wound infection models, to dissect its healing mechanisms under pathological circumstances more precisely.

In terms of scar formation, which is a natural outcome towards the end of normal wound healing, a complex series of interactions between cells, cytokines, growth factors, proteases and their inhibitors and ECM components are indispensable before a wound progresses to become a fibrous scar. Interestingly, Scharstuhl et al. ${ }^{36}$ reported that curcumin treatment at high doses induces fibroblast apoptosis through the generation of reactive oxygen species. They proposed that it may provide a novel way to modulate pathological scar formation through fine-tuning regulation of antioxidants, heme oxygenase activity and its effector molecules. As curcumin is also reported to exert anti-oxidant effects, more work is needed to further elucidate the complicated biological foundation for these findings.

Preclinical evaluation of acute wound healing includes animal models of full thickness skin defects, usually in the mouse and rat, ${ }^{37,38}$ but also in large animals such as porcine. ${ }^{39}$ Here we used rat skin defects to answer questions regarding the biocompatibility of the engineered material and, more importantly, to help elucidate the potential mechanisms of curcumin for accelerated wound healing before putting them into a clinical setting. However, as a looseskinned animal, the rat does not perfectly mimic human skin wound healing mostly due to post-wounding contracture caused by an extensive subcutaneous striated muscle layer (panniculus carnosus), which is largely absent in humans. ${ }^{40}$ Our observations, however, showed a positive effect of curcumin mainly in the later stages of wound healing, so this limitation of the model is not as pronounced in this case. The improved healing process in this model also provides sound evidence of the enhanced bioavailability of curcumin from our nanoformulation. It is worth mentioning that this simply engineered Cc/Glt NM may be well suited for future biological studies of curcumin, both in vitro and in vivo, without the fear of possible toxicity by using organic solvents such as dimethyl sulfoxide for the enhancement of curcumin's aqueous solubility, which used to be very common.

In conclusion, we show here a simple approach to engineer a smart integrated system that can act as a mechanically competent carrier that can topically deliver bioactive curcumin to wounds and thereby improve wound healing.

\section{CONFLICT OF INTEREST}

The authors declare no conflict of interest.

\section{ACKNOWLEDGEMENTS}

XD was supported by a scholarship from the China Scholarship Council and John Constable Fellowship from the American Association of Plastic Surgeons (2014). We acknowledge the support of the TUM's Faculty Graduate Center
Medical Life Science and Technology. We thank Dr Mohit Chhaya from Queensland University of Technology for consultations and help with statistics. We also thank Professor Cory Sandone from the department of art as applied to medicine, Johns Hopkins University School of Medicine, and Ms Wenjing Wu from Memorial Sloan-Kettering Cancer Center for useful consultations regarding scientific illustrations.

Author contributions: $\mathrm{XD}$ : design of in vitro and in vivo experiments, data analysis, writing of the paper. JL, HZ and JW: in vitro experiments, analysis of the data. UH: scanning electron microscopy analysis. SS and CP: fluorescent and polarized microscopy analysis. YS: animal experiments. H-GM: critically reviewed the manuscript. AFS: designed the study with $\mathrm{XD}$, analyzed the data and revised the manuscript.

1 Demidova-Rice, T. N., Hamblin, M. R. \& Herman, I. M. Acute and impaired wound healing: pathophysiology and current methods for drug delivery, part 1: normal and chronic wounds: biology, causes, and approaches to care. Adv. Skin Wound Care 25 304-314 (2012).

2 Sabale, P., Bhimani, B., Prajapati, C. \& Sabalea, V. An overview of medicinal plants as wound healers. J. Appl. Pharm. Sci. 2, 143-150 (2012).

3 Prasad, S. \& Aggarwal, B. B. in Source Herbal Medicine: Biomolecular and Clinical Aspects (eds Benzie, I.F.F. \& Wachtel-Galor, S.) 2nd edn, Chapter 13 (CRC Press/Taylor \& Francis: Boca Raton, FL, 2011).

4 Naksuriya, O., Okonogi, S., Schiffelers, R. M. \& Hennink, W. E. Curcumin nanoformulations: a review of pharmaceutical properties and preclinical studies and clinical data related to cancer treatment. Biomaterials 35, 3365-3383 (2014).

5 Akbik, D., Ghadiri, M., Chrzanowski, W. \& Rohanizadeh, R. Curcumin as a wound healing agent. Life Sci. 116, 1-7 (2014).

6 Anand, P., Kunnumakkara, A. B., Newman, R. A. \& Aggarwal, B. B. Bioavailability of curcumin: problems and promises. Mol. Pharm. 4, 807-818 (2007).

7 Sell, S. A., Wolfe, P. S., Garg, K., McCool, J. M., Rodriguez, I. A. \& Bowlin, G. L. The use of natural polymers in tissue engineering: a focus on electrospun extracellular matrix analogues. Polymers 2, 522-553 (2010).

8 Leung, V., Hartwell, R., Yang, H., Ghahary, A. \& Ko, F. Bioactive nanofibres for wound healing applications. J. Fiber Bioeng. Informatics 4, 1-14 (2011).

9 Welz, M. M. \& Ofner, C. M. Examination of self-crosslinked gelatin as a hydrogel for controlled release. J. Pharm. Sci. 81, 85-90 (1992).

10 Vogel, B., Siebert, H., Hofmann, U. \& Frantz, S. Determination of collagen content within picrosirius red stained paraffin-embedded tissue sections using fluorescence microscopy. MethodsX 2, 124-134 (2015).

11 Sun, X. Z., Williams, G. R., Hou, X. X. \& Zhu, L. M. Electrospun curcumin-loaded fibers with potential biomedical applications. Carbohydrate Polymers 94, 147-153 (2013).

12 Darby, I. A., Laverdet, B., Bonte, F. \& Desmouliere, A. Fibroblasts and myofibroblasts in wound healing. Clin. Cosmet Investig. Dermatol. 7, 301-311 (2014).

13 Shao, S., Cai, W., Sheng, J. \& Yin, L. Role of SDF-1 and Wnt signaling pathway in the myocardial fibrosis of hypertensive rats. Am. J. Transl. Res. 7, 1345-1356 (2015).

14 Kabashima, K., Sakabe, J., Yoshiki, R., Tabata, Y., Kohno, K. \& Tokura, Y. Involvement of Wnt signaling in dermal fibroblasts. Am. J. Pathol. 176, 721-732 (2010).

15 MacDonald, B. T., Tamai, K. \& He, X. Wnt/beta-catenin signaling: components, mechanisms, and diseases. Dev. Cell 17, 9-26 (2009).

16 Wood, S., Jayaraman, V., Huelsmann, E. J., Bonish, B., Burgad, D., Sivaramakrishnan, G., Qin, S., DiPietro, L. A., Zloza, A., Zhang, C. \& Shafikhani, S. H. Pro-inflammatory chemokine CCL2 (MCP-1) promotes healing in diabetic wounds by restoring the macrophage response. PLoS ONE 9, e91574 (2014).

17 Deshmane, S. L., Kremlev, S., Amini, S. \& Sawaya, B. E. Monocyte chemoattractant protein-1 (MCP-1): an overview. J. Interferon Cytokine Res. 29, 313-326 (2009).

18 Gupta, S. C., Patchva, S. \& Aggarwal, B. B. Therapeutic roles of curcumin: lessons learned from clinical trials. AAPS J. 15, 195-218 (2013).

19 Chiou, W. L. \& Riegelman, S. Pharmaceutical applications of solid dispersion systems. J. Pharm. Sci. 60, 1281-1302 (1971).

20 Husain, M. \& Khan, Z. H. Advances in Nanomaterials (Springer: India, 2016).

21 Wood, G. C. The formation of fibrils from collagen solutions. 3. Effect of chondroitin sulphate and some other naturally occurring polyanions on the rate of formation. Biochem. J. 75, 605-612 (1960).

22 Abrigo, M., McArthur, S. L. \& Kingshott, P. Electrospun nanofibers as dressings for chronic wound care: advances, challenges, and future prospects. Macromol. Biosci. 14 772-792 (2014).

23 Bolognia, J. L., Jorizzo, J. L. \& Schaffer, J. V. Dermatology, (Elsevier Health Sciences: UK, 2012).

24 Kabashima, K., Sakabe, J.-I., Yoshiki, R., Tabata, Y., Kohno, K. \& Tokura, Y Involvement of Wht signaling in dermal fibroblasts. Am. J. Pathol. 176, 721-732 (2010).

25 Qiao, L., Xu, Z. L., Zhao, T. J., Ye, L. H. \& Zhang, X. D. Dkk-1 secreted by mesenchymal stem cells inhibits growth of breast cancer cells via depression of Wnt signalling. Cancer Lett. 269, 67-77 (2008). 
26 Koch, S., Capaldo, C. T., Samarin, S., Nava, P., Neumaier, I., Skerra, A., Sacks, D. B., Parkos, C. A. \& Nusrat, A. Dkk-1 inhibits intestinal epithelial cell migration by attenuating directional polarization of leading edge cells. Mol. Biol. Cell 20, 4816-4825 (2009).

27 Seifert, O., Söderman, J., Skarstedt, M., Dienus, O. \& Matussek, A. Increased expression of the Wnt signalling inhibitor Dkk-1 in non-lesional skin and peripheral blood mononuclear cells of patients with plaque psoriasis. Acta Derm. Venereol. 95 407-410 (2015).

28 Glantschnig, H., Hampton, R. A., Lu, P., Zhao, J. Z., Vitelli, S., Huang, L., Haytko, P., Cusick, T., Ireland, C. \& Jarantow, S. W. Generation and selection of novel fully human monoclonal antibodies that neutralize Dickkopf-1 (DKK1) inhibitory function in vitro and increase bone mass in vivo. J. Biol. Chem. 285, 40135-40147 (2010).

29 Niida, A., Hiroko, T., Kasai, M., Furukawa, Y., Nakamura, Y., Suzuki, Y., Sugano, S. \& Akiyama, T. DKK1, a negative regulator of Wnt signaling, is a target of the $\beta$-catenin/ TCF pathway. Oncogene 23, 8520-8526 (2004).

30 Donadelli, R., Abbate, M., Zanchi, C., Corna, D., Tomasoni, S., Benigni, A., Remuzzi, G. \& Zoja, C. Protein traffic activates NF-kB gene signaling and promotes MCP-1-dependent interstitial inflammation. Am. J. Kidney Dis. 36, 1226-1241 (2000).

31 Trengove, N. J., Stacey, M. C., MacAuley, S., Bennett, N., Gibson, J., Burslem, F., Murphy, G. \& Schultz, G. Analysis of the acute and chronic wound environments: the role of proteases and their inhibitors. Wound Repair Regen. 7, 442-452 (1999).

32 Yamamoto, T., Eckes, B., Mauch, C., Hartmann, K. \& Krieg, T. Monocyte chemoattractant protein-1 enhances gene expression and synthesis of matrix metalloproteinase1 in human fibroblasts by an autocrine IL-1 alpha loop. J. Immunol. 164 6174-6179 (2000).

$33 \mathrm{Kim}, \mathrm{S}$. Y., Jung, S. H. \& Kim, H. S. Curcumin is a potent broad spectrum inhibitor of matrix metalloproteinase gene expression in human astroglioma cells. Biochem. Biophys. Res. Commun. 337, 510-516 (2005).

34 Jerah, A., Hobani, Y., Kumar, B. V. \& Bidwai, A. Curcumin binds in silico to anti-cancer drug target enzyme MMP-3 (human stromelysin-1) with affinity comparable to two known inhibitors of the enzyme. Bioinformation 11, 387-392 (2015).
35 Ahmad, A., Sayed, A., Ginnebaugh, K. R., Sharma, V., Suri, A., Saraph, A., Padhye, S. \& Sarkar, F. H. Molecular docking and inhibition of matrix metalloproteinase-2 by novel difluorinatedbenzylidene curcumin analog. Am. J. Transl. Res. 7, 298-308 (2015)

36 Scharstuhl, A., Mutsaers, H. A., Pennings, S. W., Szarek, W. A., Russel, F. G. \& Wagener, F. A. Curcumin-induced fibroblast apoptosis and in vitro wound contraction are regulated by antioxidants and heme oxygenase: implications for scar formation. J. Cell Mol. Med. 13, 712-725 (2009).

37 Zhang, X., Xu, R., Hu, X., Luo, G., Wu, J. \& He, W. A systematic and quantitative method for wound-dressing evaluation. Burns Trauma 3, 1 (2015).

38 Matsui, R., Osaki, K., Konishi, J., Ikegami, K. \& Koide, M. Evaluation of an artificial dermis full-thickness skin defect model in the rat. Biomaterials 17, 989-994 (1996).

39 Branski, L. K., Mittermayr, R., Herndon, D. N., Norbury, W. B., Masters, O. E., Hofmann, M., Traber, D. L., Redl, H. \& Jeschke, M. G. A porcine model of full-thickness burn, excision and skin autografting. Burns 34, 1119-1127 (2008).

40 Dorsett-Martin, W. A. Rat models of skin wound healing: a review. Wound Repair Regen. 12, 591-599 (2004).

(i) This work is licensed under a Creative Commons Attribution 4.0 International License. The images or other third party material in this article are included in the article's Creative Commons license, unless indicated otherwise in the credit line; if the material is not included under the Creative Commons license, users will need to obtain permission from the license holder to reproduce the material. To view a copy of this license, visit http:// creativecommons.org/licenses/by/4.0/

(C) The Author(s) 2017 